\title{
Fluid-structure interaction-induced oscillation of flexible structures in laminar and turbulent flows
}

\author{
Jorge Pereira Gomes $\dagger$ and H. Lienhart \\ Institute of Fluid Mechanics and Erlangen Graduate School in Advanced Optical Technologies, \\ University of Erlangen-Nuremberg, Cauerstrasse 4, D-91058 Erlangen, Germany
}

(Received 29 March 2012; revised 6 September 2012; accepted 23 October 2012)

Self-excitation of the motion of a structure has become a prominent aspect of engineering projects over recent years as designers are using materials at their limits, causing structures to become progressively lighter, more flexible and, therefore, prone to vibrate. Stimulated by the increasing interest in fluid-structure interaction (FSI) problems, this study investigated the instability and consequent FSI-induced self-excited oscillation of flexible structures in uniform flows at Reynolds numbers between 10 and $1.69 \times 10^{5}$. The investigations were performed in both water and a highly viscous syrup $\left(v=1.64 \times 10^{-4} \mathrm{~m}^{2} \mathrm{~s}^{-1}\right)$ and considered three structures of different geometries. The results were conclusive in showing that the motion of the structure was characterized by a sequence of oscillation modes as a function of the characteristics of the structure and flow properties. In addition, it was possible to identify the self-excitation mechanisms as being of the instability-induced excitation (IIE) or movement-induced excitation (MIE) types. IIE was observed to be the most dominant mechanism of excitation at lower velocities and it was defined by a direct relation between the flow fluctuation and natural frequencies of the structure. For that reason, IIE was strongly determined by the geometry of the front body of the structure. At higher velocities, the amplitudes of the flow disturbances generated by the structure movement increased and excitations of the MIE type became predominant for all structures. The MIE mechanism was found to be weakly influenced by the shape of the structure but very sensitive to its dynamic characteristics and to the properties of the fluid, especially the Reynolds number.

Key words: aerodynamics, fluid-structure interactions, vortex instability

\section{Introduction}

The excitation, and posterior amplification, of a structure and fluid system oscillation can be described, as a first approach to the problem, as follows. If a structure deforms, its orientation to the flow changes and as a result there is a change in the fluid forces acting upon the surface of the structure. The new set of fluid forces determines a new deformation of the structure. As soon as this coupled mechanism is initiated, as a result of any initial flow or movement instability, the damping imposed by the fluid can become negative as a result of different mechanisms 
by which energy is transferred from the fluid to the structure. Then, whether the oscillation is damped out or amplified is just a matter of the sign of the net damping coefficient. For lightly damped structures, the fluid damping becomes dominant and the coupled fluid and structure movement may become self-excited. Depending on whether the fluctuation of the flow or the additional velocity components introduced by the movement of the structure play the significant role in the initial excitation process, the excitation is either called flow-induced excitation or movement-induced excitation (MIE). In the case of flow-induced excitation, one can further distinguish between the extraneously-induced excitation (EIE) and instability-induced excitation (IIE) (Naudascher \& Rockwell 1991, 1993).

EIE is brought about by fluctuations of the flow velocity, or pressure, that are independent of any flow instability originated by the structure or structure motion except for added mass and fluid damping effects. The fluid forces acting upon the structure are mostly random within this excitation category, but can also be periodic, depending on whether the fluctuation of the approaching flow is periodic or not. This is the case, for example, for a structure excited by vortices shed from an upstream obstacle.

Because the excitation forces are independent of the movement and position of the structure, EIE excited oscillations cannot be considered a real case of a fluid-structure interaction (FSI) problem. Instead, EIE must be treated as forced oscillation.

IIE is caused by an instability in the flow which gives rise to flow fluctuations if a certain threshold value of the flow velocity is reached. As a rule, this instability is intrinsic to the flow created by the structure and initiates a transfer of energy even in the cases when the structure is not moving. Examples of flow instabilities are the alternate vortex shedding formation in the wake past a bluff body or impinging shear layers in jets. Depending on the control and amplification mechanism affecting the instability, the fluctuations, and the forces they generate, can become well correlated and close to the natural frequency of the structure so they can lead to large-amplitude movements.

Therefore, one may assume that this type of excitation occurs in a finite, small range of flow velocities in which the flow fluctuation triggered by the structure is in resonance with the fundamental value, or higher harmonic components, of a natural frequency of the structure. This corresponds to the condition

$$
\left(U_{r}\right)_{N, n}^{I I E}=\frac{U}{f_{N} D} \approx \frac{1}{n S t} .
$$

In the resonance range, the main feature of the FSI is the amplification of the excitation force (because of the negative damping) and the 'locking-in' of the frequency of the fluid to that of the structure frequency. In addition to showing that the fluid damping becomes negative close to resonance, Sarpkaya (1978) also argued that it is reasonable to assume that the net effect of the fluid forces in phase with the structure acceleration is, on average, zero, i.e. the fluid stiffness balances the added mass. Hence, no changes in the elastic-dynamic characteristics of the structure are expected. In other words, the global dynamic response of the oscillating system approaches very closely that of the structure alone within the IIE resonance range.

As suggested by (1.1), the vortex-shedding frequency for the stationary structure, commonly given in terms of the Strouhal number $S t$, plays an important role in the IIE mechanism. The Strouhal number depends on a large number of factors. Among all of these factors, the Reynolds number and the shape of the structure are of major importance. An insight into the influence of the shape of the structure in the Strouhal 
number is given here by the analysis of the slenderness ratio $L / D$ for cylinders with rectangular cross-section aligned with the approaching flow. This influence was studied for example, by Nakamura, Ohya \& Tsuruta (1991) $(R e=1000)$, Knisely (1990) $(720 \leqslant R e \leqslant 31000)$ and Parker \& Welsh (1983) $(17000 \leqslant R e \leqslant 35000)$. From the results, one may conclude that the excitation of most common rectangular structures (with slenderness ratios between 3 and 16 for laminar flows and between 2 and 16 for turbulent flows) is associated with the separated shear layers from the leading edges that roll up into vortices and impinge the surface of the structure. The impinging leading-edge vortex (ILEV) excitation is controlled by the feedback of pressure fluctuations resulting from the interaction of the flow vorticity with the solid boundaries. As they travel upstream and act on the source of the flow instabilities, these pressure fluctuations trigger the development of new flow fluctuations which, in turn, are amplified and produce pressure fluctuations through the flow-boundary interaction, resulting in a resonance process.

In addition to the separated vortices from the leading edge, which play the main role in this excitation mechanism, the flow pattern around the structure is characterized by unsteady vortices generated in the wake near the trailing edge by secondary motions. Thus, excitation of the ILEV type is expected to occur when the separated vortices from the leading edge grow along the surface of the structure and interact constructively with the secondary vortices at the trailing edge to form a regular vortex formation further downstream of the structure.

According to Shiraishi \& Matsumoto (1983), for structures in pure heaving oscillations, the secondary vortices at the trailing edge are generated in phase with the leading edge vortices from the opposite side of the structure. They concluded that the constructive interaction of the two types of vortices and consequently the ILEV excitation occurs when the separated vortices from the leading edge reach the trailing edge after $n(n=1,2, \ldots)$ oscillation cycles. Based on their conclusions, the resonance condition for the resonance with ILEV can be defined empirically just as a function of the structure slenderness ratio as

$$
\left(U_{r}\right)_{n}^{I I E} \approx \frac{1}{n 0.6} \frac{L}{D} .
$$

In contrast, for rectangular cylinders with a rotational degree of freedom (DOF) Shiraishi \& Matsumoto (1983) showed that the secondary vortices at the trailing edge are generated in phase with the leading edge vortices of the same side of the structure. In this case, to build a constructive interaction between the two types of vortices, the separated vortices from the leading edge ought to reach the structure trailing edge after $(2 n-1) / 2(n=1,2, \ldots)$ oscillation cycles. These conclusions are supported by the results of Komatsu \& Kobayashi (1980). They plotted the development of the vortex pattern and pressure distribution along a rectangular cylinder in cross-flow undergoing oscillations at a velocity slightly higher than the critical velocity value.

Thus, for a structure with a rotational DOF, the ILEV resonance condition expressed by (1.2) assumes the form

$$
\left(U_{r}\right)_{n}^{I I E} \approx \frac{2}{0.6(2 n-1)} \frac{L}{D} .
$$

According to Shiraishi \& Matsumoto (1983), both (1.2) and (1.3) hold for the fundamental $(n=1)$ and subsequent superharmonic ILEV resonance $(n=2)$. However, other authors have also found superharmonic resonances of higher order. 
Finally, MIE is characterized by fluctuating forces that arise from the movement of the structure itself. Whenever the structure is accelerated in the fluid, an unsteady flow is induced that alters the fluid forces acting upon the structure. If this change in the fluid load leads to negative damping and to transfer of energy from the fluid to the moving structure, the MIE mechanism starts. In this process, the forces that are responsible for the excitation are inherently linked to the movement of the structure and disappear if the structure comes to rest. The assessment of MIE is usually done by classifying the mechanism into four different subcategories: MIE independent of coupling, MIE involving mode coupling, MIE involving coupling with flow pulsation and MIE involving multiple-body coupling.

In the case of MIE independent of coupling, a single bending mode of the structure is sufficient to change the fluid forces in such a way that they produce positive work on the moving structure and energize the same bending mode. An example of this subcategory is the galloping found in short bluff bodies in cross-flows. In the subcategory of MIE involving mode coupling, two or more bending modes are involved. Even though each bending mode is free from MIE independent of coupling, it can happen that the movement associated with one mode produced fluid forces that transfer fluid energy to another bending mode, or modes, of the structure. Whenever it occurs, this self-excited mechanism is still characterized by a time delay between the bending modes involved in the energy exchange of a quarter of the oscillation period. With a time-phase shift smaller or larger than $90^{\circ}$, the oscillating movement is characterized by destructive interactions between the bending modes involved in the structure deformation followed by energy dissipation. As a consequence of this, the self-sustained mechanism of energy exchange between the bending modes would be impossible. So, MIE involving mode coupling is expected to be of easier identification and assessment through modal analysis which provides information on both phase and amplitude of the different bending modes involved in the deformation of the structure. An example of this kind of self-excitation mechanism is the flutter instability of airplane wings in which the bending mode of the structure excites the torsional mode, and vice versa. As the name suggests, MIE involving coupling with flow pulsation occurs whenever large pulsations of the flow arise associated with the structure oscillation. Finally, in the multiple-body coupling type of MIE, the fluid forces are influenced by the motion of neighbouring bodies through fluid-dynamic coupling. In this case, one may consider the dynamic coupling between two or more close structures.

In contrast to IIE, a change in the dynamics characteristics of the oscillating system is expected to occur in association with the MIE mechanism of excitation. The fact that the fluid forces contribute with a non-zero term in phase with the acceleration of the structure contributes directly to that change. This means that the evaluation of the dynamic characteristics of the oscillating system may no longer be reduced to the dynamic analysis of the structure alone. Instead, the structure and fluid have to be considered as a single oscillating body with a distinct set of dynamic characteristics, in particular natural frequencies and oscillation modes. Owing to the need to include the fluid terms in the equation of the motion, the theoretical and empirical assessment of the MIE mechanism is much more complex than the IIE. As a result, the prediction of the MIE mechanism is still largely based on experimental model tests.

For distributed-mass systems, such as articulated and flexible structures, one has to consider that an IIE excitation occurs in association with every natural frequency of the structure, as the flow velocity increases. A similar behaviour is found for MIE. Hence, several MIE oscillation modes are excited at different critical velocities 
depending on the dynamic characteristics of the fluid-structure system. Regarding the excitation due to extraneous sources, the response of a structure is defined by the conjugation of several mechanical admittance functions (each associated with a specific bending mode).

The complexity of the FSI problems increases very rapidly with the number of DOFs of the structure. Although the excitation mechanisms present distinctive typical features, they often appear superimposed and the response of the structure has to be considered as a combination of all effects. Owing to this, published work on FSI involving flexible structures is still limited in scope and, in particular, concentrates on cases where the structures are reduced to a flat plate or prismatic cylinder. Hence, the present experimental study investigated the instability and consequent FSI-induced two-dimensional oscillation of complex flexible structures in uniform axial flows. The objects of investigation were structures of different geometries which combined, to a certain extent, the elastic behaviour of thin, flexible plates in axial flow with the dynamic behaviour of rigid cylinders in cross-flow. The investigation of the coupled FSI motion was performed based on the resulting free limit cycle oscillation (LCO) of the structures and aimed at the identification and characterization of the different oscillation modes and the mechanisms which excited them.

The tests were conducted in water and a highly viscous polyethylene glycol (PEG) syrup $\left(\mu=1.64 \times 10^{-4} \mathrm{~m}^{2} \mathrm{~s}^{-1}, \rho=1050 \mathrm{~kg} \mathrm{~m}^{-3}\right)$ and covered both turbulent and laminar flow regimes. Opting for the solution of different viscous test fluids, it was possible to control the approaching flow velocity and the Reynolds number of the tests independently. This confident solution permitted this study to account for the individual influences of the approaching flow velocity and Reynolds number on the FSI self-exciting mechanisms. The measurements included the time-phase reconstruction of the periodic deformation of the structure and measurements of the principal parameters of the resulting coupled fluid and structure movement. The results of these measurements defined the general character of the elastic-dynamic response of the structures as a function of the flow velocity and characterized in detail the most relevant oscillation modes exhibited by them. The latter were used to investigate the influence of the physical parameters both in the IIE and MIE mechanisms of excitation. Furthermore, the comparison of the results obtained in turbulent and laminar flows permitted conclusions to be drawn about the effects of the Reynolds number in the FSI mechanisms.

\section{Experimental procedure}

\subsection{Experimental apparatus}

The investigations were performed in a vertical, closed-loop tunnel using two different liquids as the test fluid. It had a $180 \mathrm{~mm} \times 240 \mathrm{~mm}$ cross-sectional area and a total length of $338 \mathrm{~mm}$. As a direct consequence of the vertical orientation of the tunnel, the gravity force was aligned with the flow. In this way, the gravity force did not introduce any asymmetry in the experiments. The structure models were mounted $55 \mathrm{~mm}$ downstream of the inlet plane of the test section.

For the investigations in the laminar regime, the tunnel was operated using a PEG PG-12000 syrup as the test fluid. The measurements were performed at $25^{\circ} \mathrm{C}$ within an uncertainty of $0.5^{\circ} \mathrm{C}$. For this temperature range, the kinematic viscosity and density could be considered constant and equal to $1.64 \times 10^{-4} \mathrm{~m}^{2} \mathrm{~s}^{-1}$ and $1050 \mathrm{~kg} \mathrm{~m}^{-3}$, respectively. For the investigations in the turbulent regime, water was used as the test fluid $\left(v=0.97 \times 10^{-6} \mathrm{~m}^{2} \mathrm{~s}^{-1}, \rho=998 \mathrm{~kg} \mathrm{~m}^{-3}\right)$. 
(a)
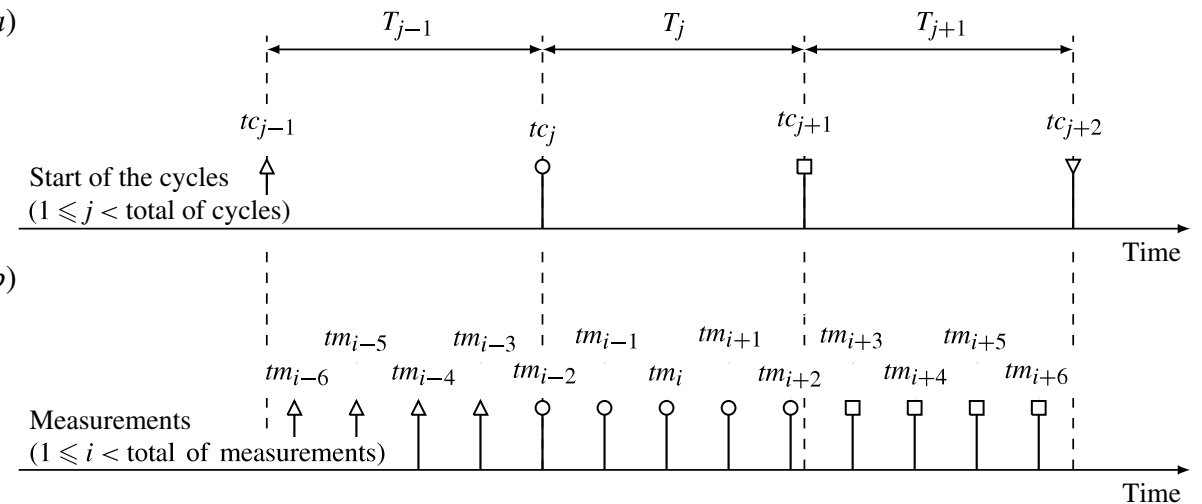

$(c)$

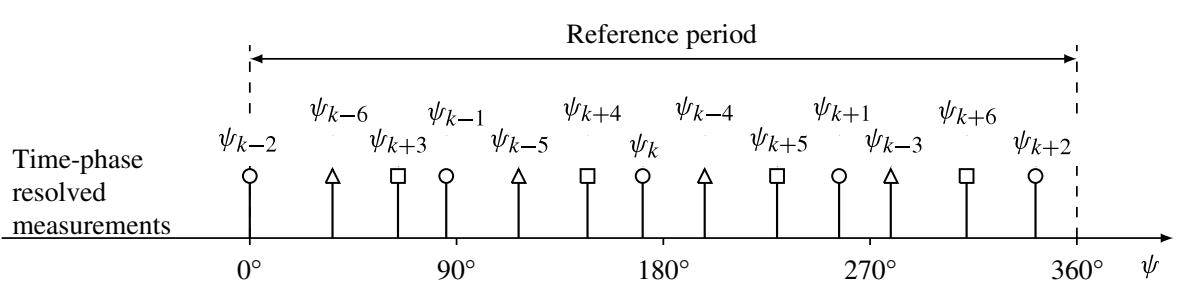

FIGURE 1. Time-phase resolved measurements reconstruction scheme (the symbols identify the motion period in which the measurements were acquired).

The deformation of the structure was measured using a stereo pattern recognition (SPR) system. Two synchronized cameras were mounted in a stereoscopic arrangement to acquire images of the flexible structure illuminated by two laser light sheets mounted perpendicular to the structure from each side of the test section. The quantitative analysis was performed in the MATLAB workspace after the acquisition of the images by a script developed specifically for the task. The software analysed and compared the images from both sides of the structure and detected the line resulting from the intersection of the laser sheet and the structure. The recognition of the reflection line, and posterior reconstruction of the structure deformation, was performed in the mathematical space, based on calibrated reference images. Opting for such a solution, an improved spatial resolution of the measurements of $7.53 \mathrm{CCD}$ pixel $\mathrm{mm}^{-1}$ was achieved.

The measurement results were reconstructed in the time-phase space introducing the time-phase angle $\psi_{k}$ based on the recorded events time information (see figure 1a,b) according to the following expression:

$$
\psi_{k}=\delta_{k i} P_{i j} \frac{t m_{i}-t c_{l} P_{i l}}{t c_{j+1}-t c_{j}} 360^{\circ},
$$

where $P_{i j}$ is the position tensor and contains information on the motion cycle in which the measurement was acquired. The tensor is defined as

$$
P_{i j}= \begin{cases}1 & \text { if } t c_{j+1}>t m_{i} \geqslant t c_{j} \\ 0 & \text { otherwise. }\end{cases}
$$

The reconstruction of results was performed using a time-phase resolution of $2.5^{\circ}$ associated with an uncertainty of $0.5^{\circ}$. The reference period for each test case was 
(a)

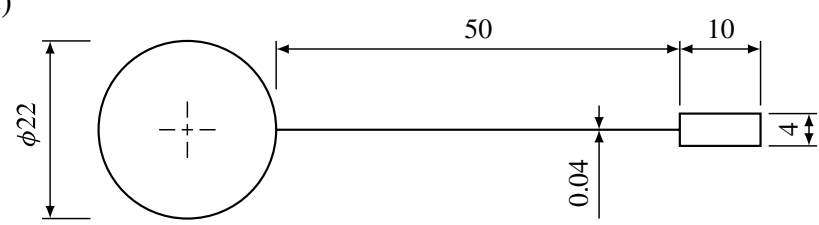

(b)

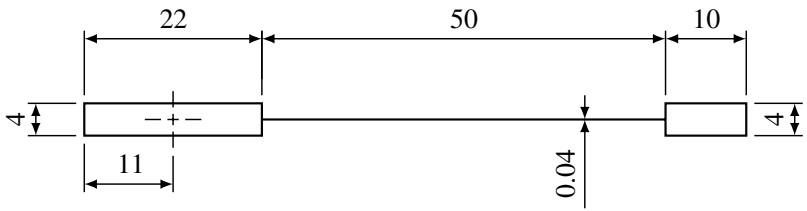

(c)

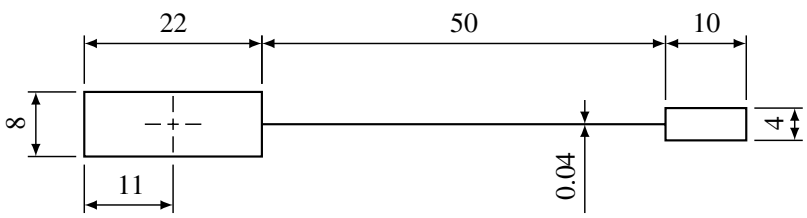

FIGURE 2. Geometry of the flexible structure models (dimensions in millimetres): (a) F2M.CH; (b) F2M.P4; (c) F2M.P8.

calculated as the mean value of all acquired swivelling periods $T_{j}$ and the maximum root-mean-square (r.m.s.) value associated with its fluctuation was measured to be $1.4 \%$.

A complete description of the test facility and measurement techniques employed in this study are given elsewhere (Gomes \& Lienhart 2010a,b).

\subsection{Definition of the models}

Three different flexible structure models were considered and their design took into account five principal requirements: (i) symmetry and reproducibility of the structure movement; (ii) two-dimensionality of the structure deformation; (iii) moderate oscillation frequencies; (iv) significant excursion of the structure, i.e. large deformations; and (v) linear material properties.

The overall length of the structures was defined as $82 \mathrm{~mm}$ and the spanwise dimension was chosen to be $177 \mathrm{~mm}$ to match the dimensions of the test section and consequently to guarantee the two-dimensionality of the tests. To allow for neglecting the effects of the flow blockage on the resulting motion, the maximum thickness of the structure was defined as $22 \mathrm{~mm}$. In this way, the ratio between the structure width and the width of the test section was maintained smaller than 0.1 . This value was supported by the results presented by Richter \& Naudascher (1976) for both circular and square cylinders in confined flows.

The geometric definition of the structure models is presented in figure 2. They consisted of a $0.04 \mathrm{~mm}$ thick stainless-steel sheet attached to a front cylindrical body. At the trailing edge of the thin sheet, a rectangular mass was considered. The F2M.CH model consisted of a circular cylinder as the front body to simulate a bluff leading edge structure. On the other side, the F2M.P4 model considered a thin rectangular cylinder as the front body to mimic a slender structure. The third structure consisted 


$\begin{array}{lccc} & \text { F2M.CH } & \text { F2M.P4 } & \text { F2M.P8 } \\ \rho_{\text {front body }}\left(\mathrm{kg} \mathrm{m}^{-3}\right) & 2828 & 7800 & 7800 \\ \rho_{\text {thin sheet }}\left(\mathrm{kg} \mathrm{m}^{-3}\right) & 7855 & 7855 & 7855 \\ \rho_{\text {rear mass }}\left(\mathrm{kg} \mathrm{m}^{-3}\right) & 7800 & 7800 & 7800 \\ m(\mathrm{~g}) & 248.8 & 181.3 & 302.8 \\ I_{0}\left(\mathrm{~kg} \mathrm{~m}^{2}\right) & 2.592 \times 10^{-4} & 2.528 \times 10^{-4} & 2.588 \times 10^{-4} \\ x_{C M}(\mathrm{~mm}) & 15.20 & 20.86 & 12.49 \\ f_{0}(\mathrm{~Hz}) & 1.90 & 1.93 & 1.90 \\ f_{1}(\mathrm{~Hz}) & 5.89 & 7.82 & 5.61 \\ f_{2}(\mathrm{~Hz}) & 27.43 & 28.44 & 27.45 \\ f_{3}(\mathrm{~Hz}) & 92.73 & 93.58 & 92.75\end{array}$

TABLE 1. Property values of the flexible structure models.

of a rectangular front body and had a geometric layout that was similar in every way to that of the F2M.P4 model except for the thickness of the front rectangular cylinder. While the F2M.P4 model considered a thinner, $4 \mathrm{~mm} \times 22 \mathrm{~mm}$ rectangular front body, the F2M.P8 model considered a thicker, $8 \mathrm{~mm} \times 22 \mathrm{~mm}$ rectangular front made from the same material. This model was chosen to represent a structure consisting of a rectangular front body with the characteristics of a bluff leading edge. The thickness of the F2M.P8 model front body was chosen in order to match its dynamic properties with those of the F2M.CH model. All structures were mounted in the test section in such a way they were able to rotate freely around an axle located in the centre of geometry of the structure front body.

All models were fine machined to guarantee maximum geometric precision. The front bodies and rear mass were machined with a geometric accuracy better than $\pm 0.1 \mathrm{~mm}$. The uncertainty associated with the length of the structures was measured to be smaller than $\pm 0.2 \mathrm{~mm}$. Finally, the uncertainty associated with the thickness of the $0.04 \mathrm{~mm}$ metal sheet was defined as $\pm 0.003 \mathrm{~mm}$. No attempts were made to polish the surface of the structures beyond their machined smoothness since it has been shown that slight-to-moderate surface roughness has no significant influence in this kind of investigation (Ramberg 1983). For the range of forces acting on the flexible structures during the investigations, the mechanical behaviour of the flexible element of the structures could be considered to be linear. Tensile tests were performed with $0.04 \mathrm{~mm}$ stainless-steel sheets and the Young's modulus was measured to be $200 \mathrm{~N} \mathrm{~mm}^{-2}$ within this range of strain. The other elements of the structure, the front body and the rear mass, were considered rigid.

Table 1 summarizes the mechanical properties of the structures together with the density of the materials used in their construction.

\section{Results}

To assist the reader, the results achieved for each structure are presented here in a systematic form as follows. First, the general character of the elastic-dynamic response of the structure is described at different flow velocities up to $2 \mathrm{~m} \mathrm{~s}^{-1}$ by means of the structure movement amplitude and oscillation frequency. The error bars in the figures are a measure of the r.m.s. values associated with the cycle-to-cycle fluctuation of the measured quantities. Then, the resulting movement of the structure at selected flow velocities is described in detail. The results are presented as time-phase traces 
of the structure front body angle and trailing edge $x y$ coordinates. Simultaneously, the deformation of the structure for successive equidistant time-phase angles is shown. The resolution adopted for the representation of the latter was $30^{\circ}$ and the position of the rear mass was not displayed for clarity. Also for clarity, the results of the trailing-edge position were drawn to scale.

Concerning the accuracy of the measurements, it was difficult to estimate an unique uncertainty value for the time-phase resolved results of the structure deformation and trailing-edge position. Because the test were focused in the free oscillation of flexible structures, the cycle-to-cycle fluctuations of the structure position had to be considered in addition to the uncertainty of the measuring technique. These fluctuations were very sensitive to several factors, in particular the time-phase angle, position of the measuring point, and fluid viscosity. After the analysis of the most critical points of the structure deformation and time-phase angles, one could conclude that the maximum r.m.s. value associated with the fluctuation of the structure deformation results during the tests in laminar and turbulent regime was $\sim 0.26$ and $0.40 \mathrm{~mm}$, respectively, within a confidence interval of $90 \%$.

Finally, the results of the modal analysis are shown. This analysis was performed using the finite-element ANSYS structural mechanics computed-aided engineering (CAE) software using 2-DOF structural solid elements with realistic mechanical properties and considered the first 15 bending modes of the structures $(1 \leqslant j \leqslant 15)$. Comparison of the results obtained for different numerical models, such as rigid structural solid elements to model the rigid elements of the structures, showed that the uncertainty of the results associated with the first bending mode was close to $5 \%$. For the higher bending modes, the uncertainty of the results were negligible. The natural frequency associated with the zeroth bending mode, i.e. with the rigid body movement of the structure, was computed separately by analytical means using the following expression:

$$
f_{0}=\frac{1}{2 \pi} \sqrt{\frac{m g l}{I_{\theta}}} .
$$

The results presented in the following figures correspond to the decomposition of the time-phase resolved deformation of the structure in its individual bending modes, according to the following expression:

$$
y(\psi, x)=q_{j}(\psi) \phi_{j}(x) .
$$

The bending-mode energy was computed using the following expression

$$
E_{j}=\int_{0^{\circ}}^{360^{\circ}} q_{j}(\psi) \mathrm{d} \psi
$$

and the values were normalized to unity.

\subsection{Results in the laminar regime}

The investigations in the laminar regime were performed in a PEG syrup at a controlled temperature of $25^{\circ} \mathrm{C}$ with an uncertainty of $0.5^{\circ} \mathrm{C}$. For this range of temperatures, the liquid could be considered incompressible and Newtonian; its kinematic viscosity and density were measured to be constant at $1.64 \times 10^{-4} \mathrm{~m}^{2} \mathrm{~s}^{-1}$ and $1050 \mathrm{~kg} \mathrm{~m}^{-3}$, respectively.

The Reynolds number of the experiments, based on the kinematic viscosity of the PEG syrup and on a characteristic length equal to the overall length of the models, 

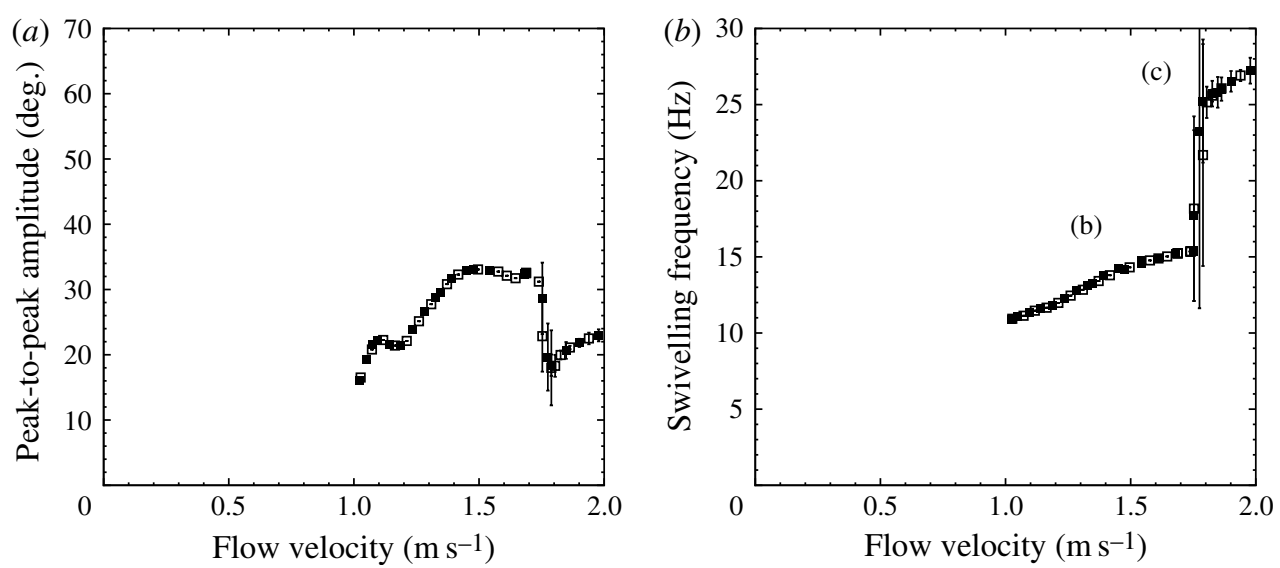

FIGURE 3. Oscillation amplitude $(a)$ and frequency $(b)$ versus flow velocity for the F2M.P4 model in laminar flows.

reached a maximum value of 1000 . For the F2M.CH model, it was also meaningful to define the characteristic length as the diameter of the front cylinder. In this case, the Reynolds number of the tests was restricted to a maximum value close to 270 .

\subsubsection{Results with the F2M.P4 model}

Figure 3 shows the elastic-dynamic response of this structure as a function of the approaching flow velocity (solid squares correspond to measurements acquired while increasing and open squares while decreasing the approaching flow velocity).

The most important aspect revealed by figure 3 was the existence of two distinctive oscillation modes. The excitation of the structure movement from rest to the first mode was observed for a flow velocity close to $1 \mathrm{~m} \mathrm{~s}^{-1}$ without evidences of hysteresis. Visualizations performed for velocities between approximately 1 and $1.7 \mathrm{~m} \mathrm{~s}^{-1}$ showed that this oscillation mode (see (b) in figure $3 b$ ) was characterized by the fact that the deformation of the structure was strongly dominated by the second bending mode of the structure. In connection to this, the movement of the rear mass was in phase opposition with the rotation of the front body. This qualitative description of the movement based on visualizations was supported by detailed measurements performed at both 1.07 and $1.45 \mathrm{~m} \mathrm{~s}^{-1}$. As far as the excitation amplitude is concerned, figure 3(a) shows two local maxima in the excitation of the structure movement. The first was registered at $\sim 1.13 \mathrm{~m} \mathrm{~s}^{-1}$ and the second at $1.5 \mathrm{~m} \mathrm{~s}^{-1}$.

On increasing the flow velocity, it was possible to observe a new oscillation mode for velocities higher than $1.76 \mathrm{~m} \mathrm{~s}^{-1}$. The excitation of the new mode (see (c) in figure $3 b$ ) resulted in a non-reproducible movement. In addition to considerable cycleto-cycle fluctuations of the motion parameters, there were intermittent periods of time in which the structure failed to cross the $x$-axis during its oscillating movement, giving the time-phase detector a chance to register double and triple oscillation time periods. For that reason, the measurement results corresponding to this oscillation mode should be interpreted with caution. The values plotted in figure 3 were averaged after filtering out all of the multiple periods. Even so, the r.m.s. associated with the fluctuation of the frequency of the structure movement at $1.92 \mathrm{~m} \mathrm{~s}^{-1}$ exceeded $7 \%$. Because of this behaviour, no detailed measurements were performed at flow velocities higher than $1.76 \mathrm{~m} \mathrm{~s}^{-1}$. Nevertheless, visualizations conducted at $1.92 \mathrm{~m} \mathrm{~s}^{-1}$ proved that the 
third bending mode of the structure was in evidence and dominated the deformation of the structure. This observation was supported by the existence of two clear nodal regions in the oscillating movement of the structure. Within this oscillation mode, the movement of the trailing edge was in phase with the rotation of the front body.

For the present structure, detailed measurements were performed for an approaching flow velocity of 1.07 and $1.45 \mathrm{~m} \mathrm{~s}^{-1}$. These two values were chosen because they were located close to the velocity of local maxima in the amplitude of the structure movement in the ascending section (see figure $3 a$ ).

Results at $1.07 \mathrm{~m} \mathrm{~s}^{-1}$

The following figures show the results for an approaching flow velocity of $1.07 \mathrm{~m} \mathrm{~s}^{-1}$, which corresponds to a Reynolds number of 535. At this velocity, the frequency of the resulting movement of the structure was measured to be $11.22 \mathrm{~Hz} \pm 0.4 \%$.

Figure 4(a) shows the time-phase trace of the angle of the front body within an oscillation period. The r.m.s. value associated with the fluctuation of this result was measured to be $0.5^{\circ}$. In the same figure, the transverse displacement of the structure trailing edge is shown. Comparison of the curves indicates that the movement of the trailing edge was in opposition with respect to the rotation of the front body. The time-phase delay between the two was measured to be approximately $250^{\circ}$.

Figure $4(b)$ contains the information about the deformation of the structure for successive instants within an oscillation period. In figure 4(c), the successive positions occupied by the trailing edge within the same time period are presented. At the beginning of the cycle, the coordinates of the trailing edge were measured to be $\left.(x ; y)\right|_{\psi=0^{\circ}}=(67.78 \mathrm{~mm} ;-9.41 \mathrm{~mm})$.

\section{Results at $1.45 \mathrm{~m} \mathrm{~s}^{-1}$}

An identical set of measurements were performed at $1.45 \mathrm{~m} \mathrm{~s}^{-1}$. At this velocity, the Reynolds number was 725 and the frequency of the structure movement was measured to be $13.78 \mathrm{~Hz} \pm 0.5 \%$.

Figure 5(a) shows the time-phase trace of the front body angle and also the transverse displacement of the structure trailing edge within an oscillation period. The r.m.s. value associated with the fluctuation of the front body angle was close to $0.5^{\circ}$, and the time-phase difference between the trailing edge and front body was measured to be approximately $225^{\circ}$, that is, the trailing edge was delayed, moving in opposition, with respect to the front body rotation.

Figure 5(b) shows the deformation of the structure for successive time-phase angles. Figure 5(c) shows the trajectory followed by the structure trailing edge within an oscillation period. At the beginning of the period, the trailing edge was registered to be at the point $\left.(x ; y)\right|_{\psi=0^{\circ}}=(64.90 \mathrm{~mm} ;-9.05 \mathrm{~mm})$.

The results obtained with the F2M.P4 model showed that both oscillation modes were triggered by the MIE mechanism and were characterized by an oscillation frequency much lower than the second natural frequency of the structure.

The deformation of the structure within the MIE oscillation mode which was excited at $\sim 1 \mathrm{~m} \mathrm{~s}^{-1}$ is presented here by the analysis of the deformation of the structure at $1.45 \mathrm{~m} \mathrm{~s}^{-1}$ (see figure 6). Because they correspond to the same oscillation mode, the analysis of the structure deformation at $1.07 \mathrm{~m} \mathrm{~s}^{-1}$ led to similar results as those shown in figure 6. In fact, the similarity between the deformation of the structure at the two different flow velocities was already proven on comparing figures $4(b)$ and $5(b)$. Figure 6 confirmed that, the oscillation mode found within 1 and $1.76 \mathrm{~m} \mathrm{~s}^{-1}$ (see (b) in figure $3 b$ ) was self-excited by the MIE mechanism involving mode coupling. 

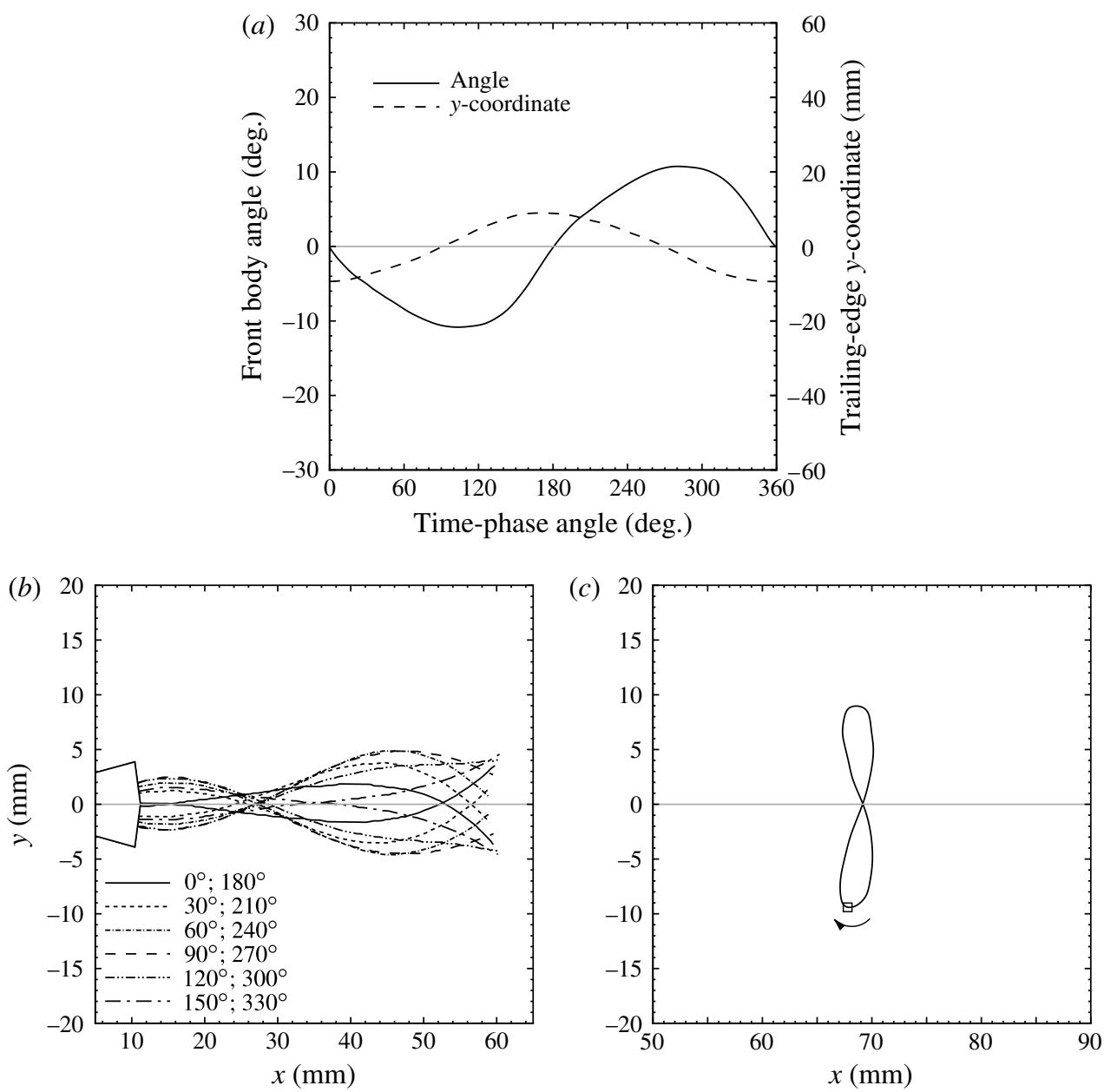

FIGURE 4. Front body angle and trailing edge $y$ coordinate $(a)$, deformation of the structure $(b)$ and trailing edge coordinates $(c)$, within a period of motion at $1.07 \mathrm{~m} \mathrm{~s}^{-1}$ in laminar flows, with the F2M.P4 model.

In this particular case, the results of the energy distribution show that all bending modes up to the third-order were significantly involved in the control of the structure deformation. Figure 6 also shows that the time-phase delay between the zeroth, first and third bending modes and the second bending mode of the structure was nearly constant. Exactly, the time-phase shift between the second and the other three modes was measured between 87 and $117^{\circ}$. All evidences indicate that the second bending mode of the structure was excited and exchanged energy with the group consisting of the rest of the significant bending modes involved in the deformation of the structure. Confirming an intrinsic characteristic of the MIE mechanism involving mode coupling, the time delay between the exciting bending mode and all of the other modes involved in the excitation was close to a quarter of the oscillation period.

One may observe that the zeroth, first and third bending mode were in phase with each other. So, no energy was exchanged between these bending modes. By this reason, this oscillation mode can also be understood as an oscillation mode triggered 

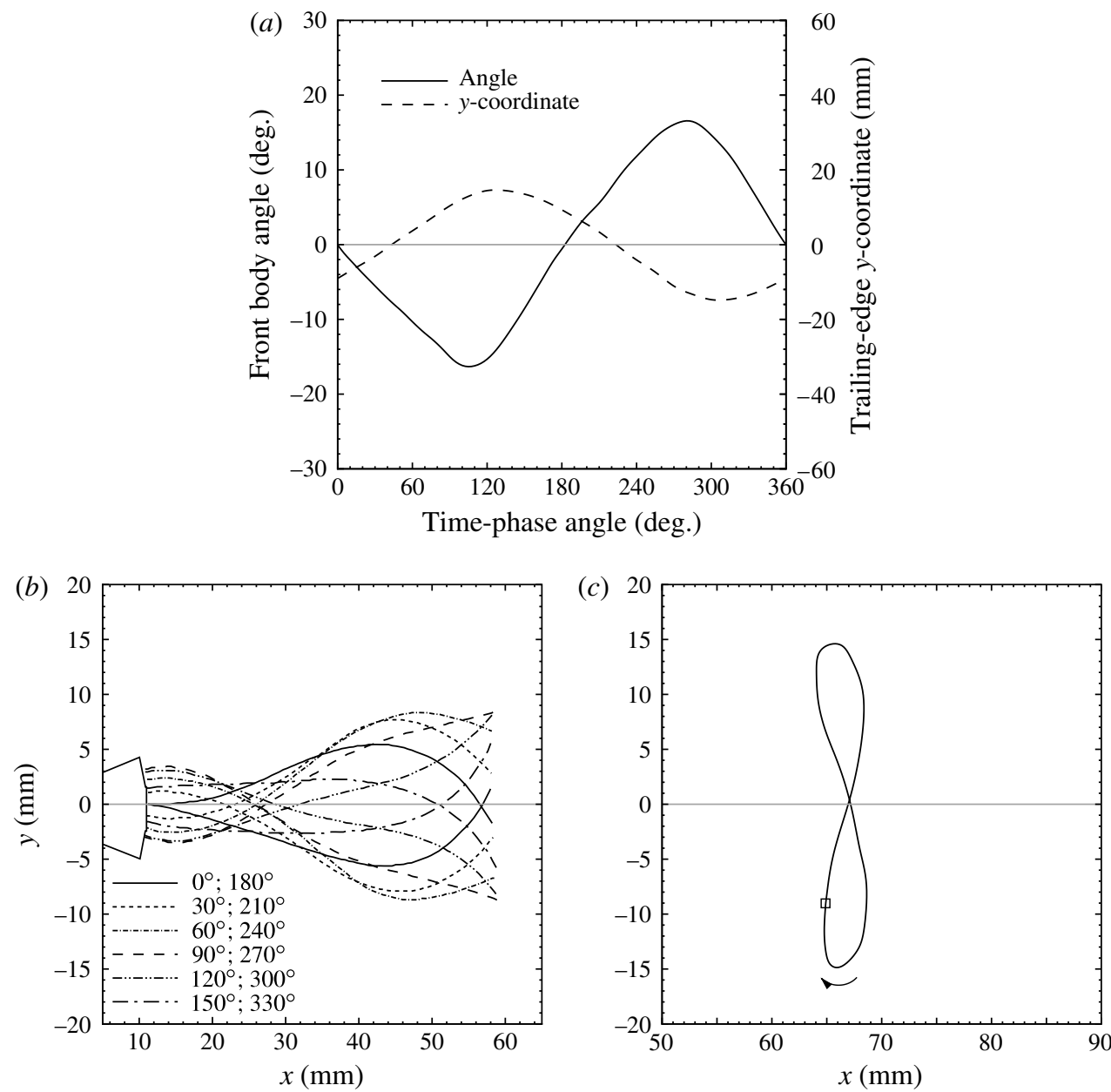

FIGURE 5. Front body angle and trailing edge $y$ coordinate (a), deformation of the structure $(b)$ and trailing edge coordinates $(c)$, within a period of motion at $1.45 \mathrm{~m} \mathrm{~s}^{-1}$ in laminar flows, with the F2M.P4 model.

by the MIE mechanism involving the coupling of just two bending modes; the second bending mode of the structure and a new bending mode resulting from the sum of the zeroth, first and third bending modes. The time delay between the latter and the second bending mode of the structure was approximately equal to a quarter of the oscillation period.

Within the oscillation mode found for velocities higher than $1.76 \mathrm{~m} \mathrm{~s}^{-1}$ (see (c) in figure $3 b$ ), a similar mechanism of excitation was found. The analysis of the deformation of the structure, based on visualizations performed at $1.92 \mathrm{~m} \mathrm{~s}^{-1}$, revealed that in this case, the third bending mode of the structure was the exciting mode of the MIE involving mode coupling and energy was exchanged between this mode and the zeroth, first and second bending modes of the structure.

About the local maxima found in the amplitude excitation of the structure at $\sim 1.13$ and $1.5 \mathrm{~m} \mathrm{~s}^{-1}$, the results showed a close connection to the local resonance phenomena involving the oscillation frequency and the natural frequency, or harmonic 

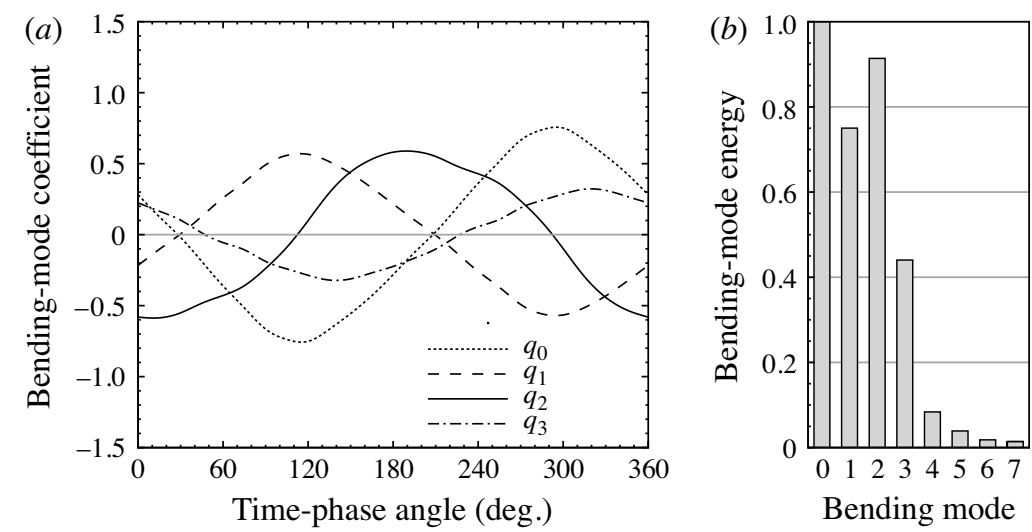

FIGURE 6. Decomposition of the F2M.P4 model deformation in the individual bending modes at $1.45 \mathrm{~m} \mathrm{~s}^{-1}$ in laminar flows.

components of the natural frequency, of the structure. Whereas the amplitude response of the structure has proved to be very sensitive to these local resonance phenomena, similar effects were not observed in the swivelling frequency of the structures.

Finally, one may observe the absence of IIE oscillation modes for the F2M.P4 model. This behaviour could be justified by the fact that the laminar vortex shedding past the cylinder is suppressed at very low Reynolds numbers. Because the critical Reynolds number for the onset of the laminar vortex shedding is similar to that for circular and rectangular cylinders, it is easy to show that at $\sim 1 \mathrm{~m} \mathrm{~s}^{-1}$, the point at which the movement of the structure was excited as a consequence of MIE mechanism, the Reynolds number of the experiment was still very low compared with the critical value $(R e \approx 47)$ to cause the shedding of laminar vortices from the rectangular front body. This argument supports the conclusion that excitations of the oscillation modes with the F2M.P4 model were both of the MIE type.

\subsubsection{Results with the F2M.CH model}

This structure considered a circular front body instead of the rectangular shape considered in the previous test case. The elastic-dynamic response of this structure is represented in figure 7 as a function of the approaching flow velocity (the arrow indicates the critical velocity value according to (1.1)).

This structure exhibited the same multi-oscillation-mode behaviour to that observed for the rectangular case. However, on comparing the two structures, there were noticeable differences. The most significant differences were related to the earlier excitation of the structure and the pronounced hysteretic region which separated the two oscillation modes exhibited by the structure. Regarding the first oscillation mode, it was observed that the velocity threshold to excite the movement of the structure varied slightly from model to model, on increasing the flow velocity. Nevertheless, in all tests it was possible to achieve a periodic oscillation for velocities higher than $0.8 \mathrm{~m} \mathrm{~s}^{-1}$. According to visualizations performed at different flow velocities, the first oscillation mode (see (a) in figure $7 b$ ) was characterized by the fact that the deformation of the structure was strongly dominated by the first bending mode of the structure. In connection with this, the movement of the rear mass was in phase agreement with the rotation of the front body. 

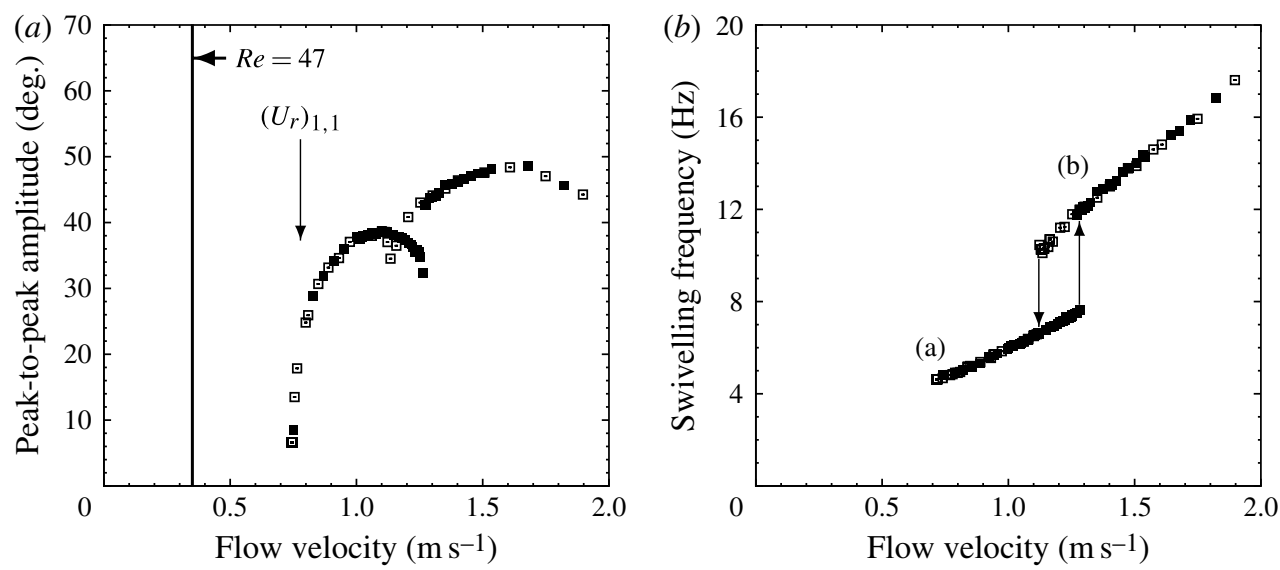

FIGURE 7. Oscillation amplitude $(a)$ and frequency $(b)$ versus flow velocity for the F2M.CH model in laminar flows.

In contrast, the second oscillation mode (see (b) in figure $7 b$ ) was characterized by the fact that the rear mass movement was in opposition to the rotation of the structure front body. At the same time, higher structure bending modes with predominance of the second were observed to control the deformation of the structure.

Both modes were characterized by a linear dependence between the resulting oscillation frequency and the approaching flow velocity. While the frequency increased monotonically with the flow velocity, the amplitude of the movement presented a local maximum for each oscillation mode, at approximately 1.1 and $1.65 \mathrm{~m} \mathrm{~s}^{-1}$, respectively.

Within the transition regime, between 1.1 and $1.3 \mathrm{~m} \mathrm{~s}^{-1}$, the structure presented a well-defined hysteretic behaviour where both oscillation modes could be observed depending on the previous frequency of the structure. At the extremes of this regime, the transition between modes was always abrupt and no transient could be measured. The same hysteretic behaviour was found at the onset of the first oscillation mode, as that the minimum velocity to maintain a self-excited structure motion was always lower that the velocity threshold to excite the structure movement from rest.

For the present structure, detailed measurements were performed at 1.07 and $1.45 \mathrm{~m} \mathrm{~s}^{-1}$. These values were located close to the velocity of maximum structure amplitude (see figure $7 a$ ), and at the same time they matched the velocity values investigated for the previous model.

Results at $1.07 \mathrm{~m} \mathrm{~s}^{-1}$

At a flow velocity of $1.07 \mathrm{~m} \mathrm{~s}^{-1}$, the Reynolds number of the test, based on the length of the model, was 535. If the front body diameter is considered as the characteristic length, the Reynolds number was approximately 145 . At this velocity, the resulting structure oscillation was measured to have a frequency equal to $6.38 \mathrm{~Hz} \pm 0.6 \%$.

Figure 8(a) shows the time-phase trace of the front body angle and transverse displacement of the structure trailing edge within an oscillation period. Comparison of the two curves in figure $8(a)$ indicates that the movement of the trailing edge was delayed, but moving in agreement, with respect to the movement of the front body. 

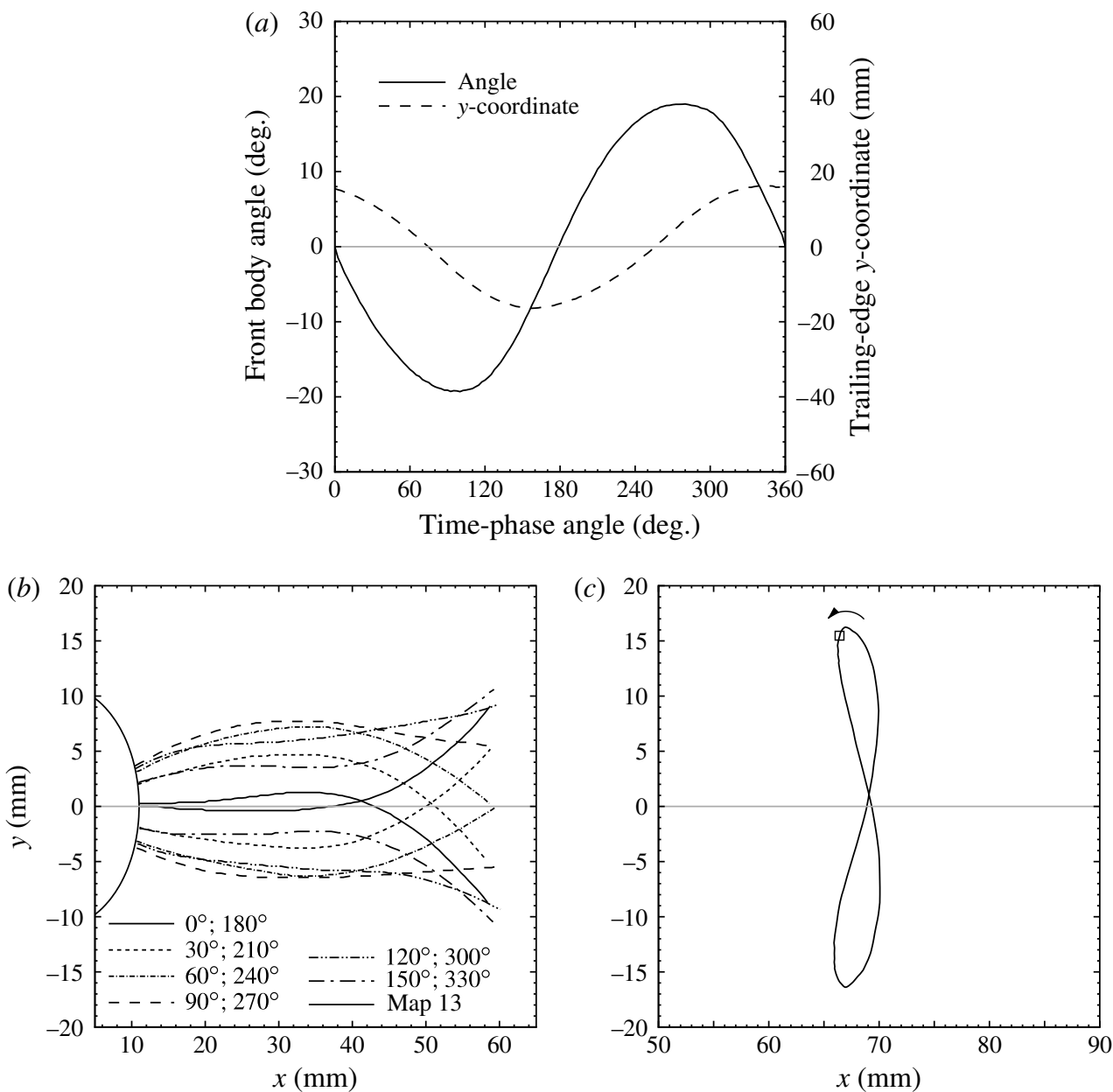

FIGURE 8. Front body angle and trailing edge $y$ coordinate $(a)$, deformation of the structure $(b)$ and trailing edge coordinates $(c)$, within a period of motion at $1.07 \mathrm{~m} \mathrm{~s}^{-1}$ in laminar flows, with the F2M.CH model.

This delay was measured to be approximately $75^{\circ}$ and the r.m.s. value associated with the fluctuation of the front body angle was measured to be less than $0.5^{\circ}$.

In figure $8(b)$, the deformation of the structure is presented for successive instants within an oscillation period. In figure $8(c)$, the trajectory followed by the trailing edge within the same period is shown. At the beginning of the oscillation period, the trailing edge was measured to be at the point $\left.(x ; y)\right|_{\psi=0^{\circ}}=(66.43 \mathrm{~mm} ; 15.46 \mathrm{~mm})$.

\section{Results at $1.45 \mathrm{~m} \mathrm{~s}^{-1}$}

A similar set of measurements were performed for an approaching flow velocity of $1.45 \mathrm{~m} \mathrm{~s}^{-1}$, which corresponded to a Reynolds number close to 725 or 195 , depending on whether it was computed based on the overall length of the model or on the diameter of the front circular body. At $1.45 \mathrm{~m} \mathrm{~s}^{-1}$, the structure exhibited a more complex and faster oscillation with a frequency equal to $13.58 \mathrm{~Hz} \pm 0.9 \%$.

Figure 9(a) shows the time-phase trace of the angle of the front body and also the transverse displacement of the trailing edge within an oscillation period. The 

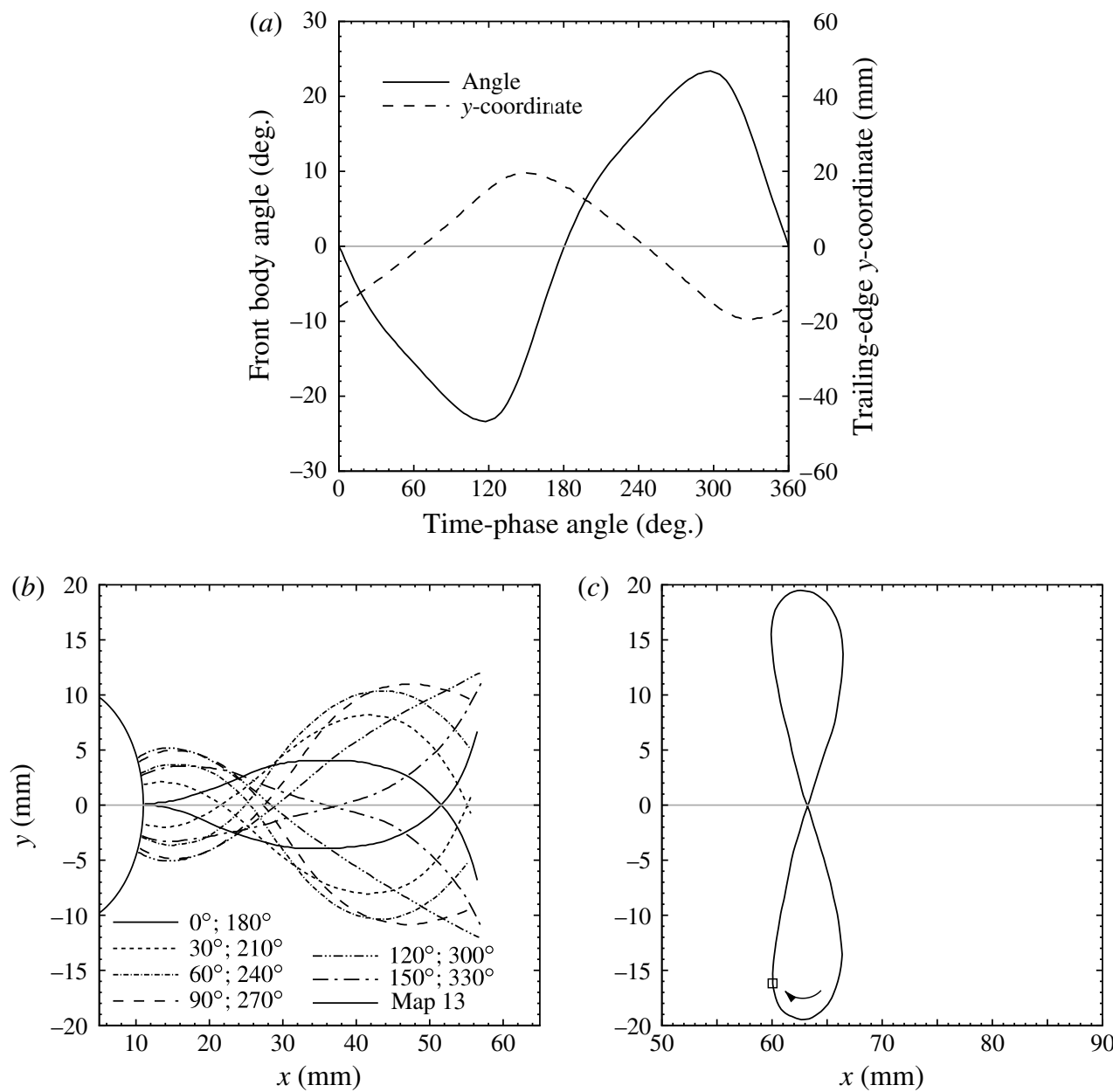

FIGURE 9. Front body angle and trailing edge $y$ coordinate $(a)$, deformation of the structure $(b)$ and trailing edge coordinates $(c)$, within a period of motion at $1.45 \mathrm{~m} \mathrm{~s}^{-1}$ in laminar flows, with the F2M.CH model.

r.m.s. value associated with the fluctuation of the front body angle was measured to be $0.5^{\circ}$. The movement of the structure trailing edge could now be considered in opposition with respect to the front body movement, with a time-phase delay of $\sim 245^{\circ}$.

Figure $9(b)$ shows the structure deformation for successive time-phase angles within an oscillation period. Figure $9(c)$ shows the successive positions occupied by the trailing edge within the same period. At the beginning of the period, the coordinates of the trailing edge were $\left.(x ; y)\right|_{\psi=0^{\circ}}=(60.06 \mathrm{~mm} ;-16.19 \mathrm{~mm})$.

The results were conclusive in showing that the main differences between the two structures were related to the fact that their movements were excited by different mechanisms of excitation. Indeed, excitation of the first oscillation mode of the F2M.CH model (see (a) in figure 7a) was ruled by the IIE mechanism. The comparison of the Strouhal number and the natural frequencies of the structure showed a strong interconnection between the classical vortex shedding triggered by 

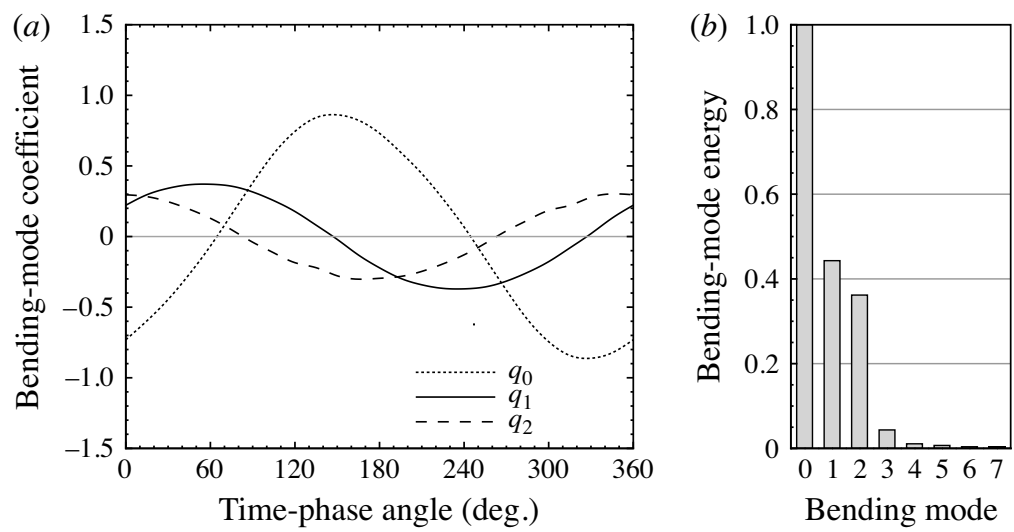

FIGURE 10. Decomposition of the F2M.CH model deformation in the individual bending modes at $1.07 \mathrm{~m} \mathrm{~s}^{-1}$ in laminar flows.

the structure front circular cylinder and the excited movement. At the onset of the movement, between 0.75 and $0.8 \mathrm{~m} \mathrm{~s}^{-1}$, equation (1.1) shows that there was a direct relation between the Strouhal number $(S t \approx 0.167)$ (Fey, Konig \& Eckelmann 1998; Williamson \& Roshko 1990; Williamson \& Brown 1998), based on the diameter of the front circular cylinder, and the first natural frequency of the structure $\left(f_{1}=5.89 \mathrm{~Hz}\right)$. The critical velocity $\left(U_{r}\right)_{1,1}$ correspondent to this situation is identified in figure $7(a)$. In other words, it shows that the flow fluctuation was in resonance with the first natural frequency of the structure.

This conclusion was confirmed by the analysis of the structure deformation measured at $1.07 \mathrm{~m} \mathrm{~s}^{-1}$. Figure 10 shows the results of the analysis of the deformation of the F2M.CH model at this velocity. It confirms the resonance of the first bending mode of the structure. The simultaneous excitation of the zeroth and second bending modes reinforces the statement that, in this case, the IIE excitation was followed by an excitation of the MIE type involving mode coupling. Both the zeroth and second bending modes are nearly in phase with each other and delayed with respect to the first bending mode by about one-quarter of the period. For example, the time-phase delay between the first and zeroth oscillation modes was measured as $97.6^{\circ}$. This indicates that after the excitation of the first bending mode of the structure through the IIE mechanism, the self-sustained MIE mechanism of transfer of energy between the first bending mode of the structure and the zeroth and second bending modes was triggered, as soon as the amplitude of the structure movement exceeded a certain threshold.

The oscillation mode exhibited by the F2M.CH model for velocities higher than approximately $1.1 \mathrm{~m} \mathrm{~s}^{-1}$ (see (b) in figure $7 b$ ) was once again triggered by the MIE mechanics and it was dominated by the second bending mode of the structure. In particular, this oscillation mode had the same characteristics as the oscillation mode exhibited by the F2M.P4 model for velocities between 1 and $1.76 \mathrm{~m} \mathrm{~s}^{-1}$. The analysis of the deformation of the F2M.CH model supports this statement. The results of this analysis for a flow velocity of $1.45 \mathrm{~m} \mathrm{~s}^{-1}$, presented in figure 11, are very similar to those in figure 6. In figure 11, the time-phase shift between the second and the other bending modes was measured between 70.3 and $114.2^{\circ}$. 

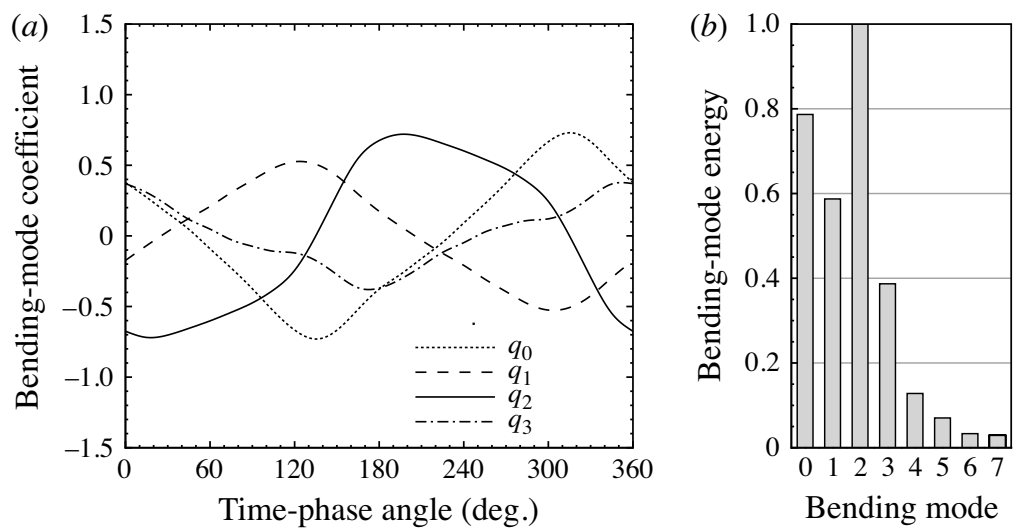

FIGURE 11. Decomposition of the F2M.CH model deformation in the individual bending modes at $1.45 \mathrm{~m} \mathrm{~s}^{-1}$ in laminar flows.

Although an IIE excitation associated with the resonance of the flow fluctuation with the first natural frequency of the structure was found, one may notice that this structure did not exhibit the zeroth oscillation mode (associated with the zeroth bending mode or rigid body movement of the structure). This oscillation mode was not observed because the Reynolds number was below the critical value for the formation of a regular vortex shedding past a circular cylinder $(R e \approx 47)$. A rough analysis of the resonance condition given by (1.1) estimated that the IIE resonance of the zeroth bending mode of the structure, if it happened, should have occurred at a velocity slightly smaller than that in which the critical Reynolds number was reached $\left(U \approx 0.35 \mathrm{~m} \mathrm{~s}^{-1}\right)$.

\subsection{Results in turbulent regime}

The investigations in the turbulent regime were performed in water at a controlled temperature of $22_{-1}^{+0}{ }^{\circ} \mathrm{C}$. For this temperature range, the kinematic viscosity and the density of the water could be considered as $0.97 \times 10^{-6} \mathrm{~m}^{2} \mathrm{~s}^{-1}$ and $998 \mathrm{~kg} \mathrm{~m}^{-3}$, respectively. Based on these values and on the overall length of the models, the maximum Reynolds number achieved during the tests was $\sim 16.9 \times 10^{4}$. For the model consisting of a circular front body, the maximum Reynolds number of the tests, based on the diameter of the front cylinder, was limited to $4.54 \times 10^{4}$.

\subsubsection{Results with the F2M.P4 model}

The elastic-dynamic response of this structure is shown in figure 12 as a function of the approaching flow velocity (solid squares correspond to measurements acquired while increasing and open squares while decreasing the approaching flow velocity).

The minimum flow velocity needed to excite the structure was measured to be $0.65 \mathrm{~m} \mathrm{~s}^{-1}$. During the excitation of the first oscillation mode (see (a) in figure 12a), the structure deformation was mostly dominated by the first bending mode of the structure. In connection with this, the movement of the trailing edge was found to be in agreement with the rotation of the front body.

The transition to a new oscillation mode was recorded at around $0.96 \mathrm{~m} \mathrm{~s}^{-1}$ and it was characterized by an abrupt change in the oscillation frequency. From the point of view of the movement amplitude, the two oscillation modes were separated by a hysteretic region centred at $\sim 1 \mathrm{~m} \mathrm{~s}^{-1}$. The new oscillation mode (see (d) in figure 12a) 

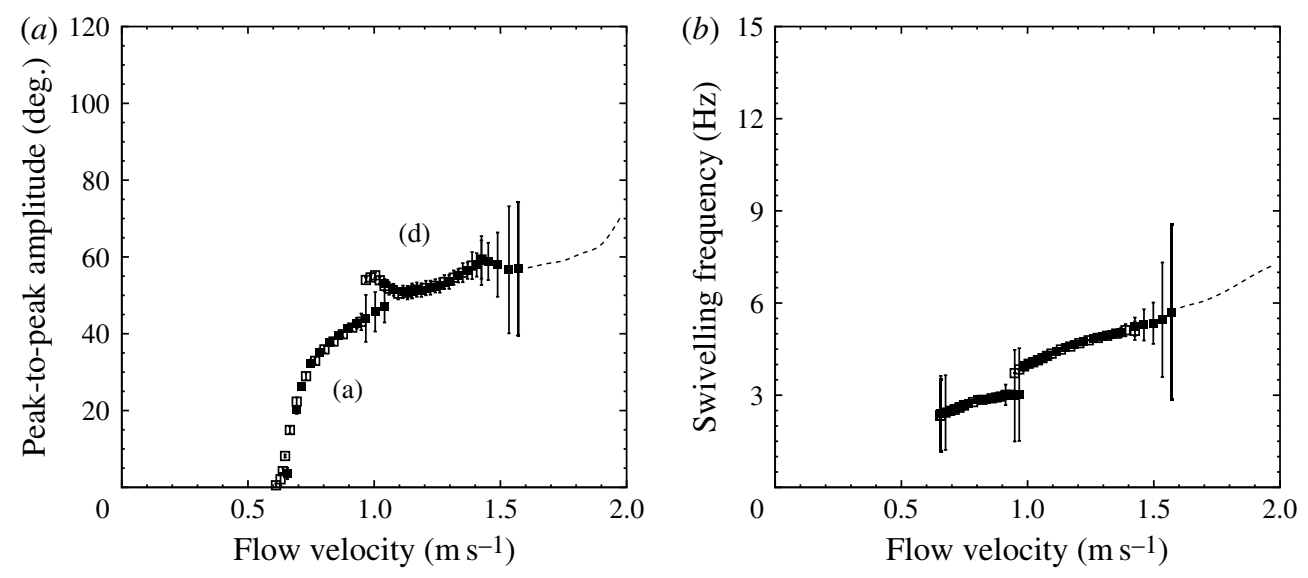

FIGURE 12. Oscillation amplitude $(a)$ and frequency $(b)$ versus flow velocity for the F2M.P4 model in turbulent flows.

was more complex and the characteristics of the structure movement proved to be unique in comparison with all of the other oscillation modes measured in both laminar and turbulent flows. This statement was supported by the observation of the structure movement at different flow velocities within the range $1.05-1.4 \mathrm{~m} \mathrm{~s}^{-1}$ and by detailed measurements performed for a constant approaching velocity of $1.22 \mathrm{~m} \mathrm{~s}^{-1}$. They were conclusive in indicating that this mode possessed the characteristics of an oscillation mode in which the deformation of the structure was dominated by the first bending mode. In agreement with this first conclusion, figure $14(a, b)$, obtained at a flow velocity of $1.22 \mathrm{~m} \mathrm{~s}^{-1}$, show no nodal regions in the deformation of the structure and prove that the trailing edge was moving in agreement with the rotation of the front body.

However, when figure $14(b)$ is analysed in more detail, it becomes clear that higher harmonic components rather than just the fundamental natural frequencies of the structure were presented in the deformation of the structure. The supremacy of the fundamental value of the first natural frequency in the structure deformation is better seen at time-phase angles of 30 and $210^{\circ}$. On the other hand, the superimposition of higher harmonics is clearly seen for $\psi=60^{\circ}$ and $\psi=240^{\circ}$. In sum, figure $14(b)$ shows that higher harmonics of the structure natural frequencies actively contributed to the control of the deformation of the structure. This situation justified the unique characteristics of the present oscillation mode. Among the unusual features, the timephase trace of the front body angle was characterized by a 'figure-of-M' shape in which two positive and two negative local maxima were identified. The shape of the trailing edge trajectory no longer assumed the classical 'figure-of-eight' shape and exhibited two singular points where a sudden reverse in the direction of the movement was observed.

Towards the upper limit of the test velocity range, the motion characteristics degraded significantly. The first signs of irregularities were observed in both oscillation frequency and amplitude at $\sim 1.4 \mathrm{~m} \mathrm{~s}^{-1}$ and the movement of the structure became totally non-symmetric and non-periodic at flow velocities higher than $1.6 \mathrm{~m} \mathrm{~s}^{-1}$. This behaviour persisted until the end of the test, at $2 \mathrm{~m} \mathrm{~s}^{-1}$, even though the structure could maintain its physical integrity. The fact that the first natural frequency of the 
structure was equal to $7.8 \mathrm{~Hz}$ inhibited a resonance of the structure and contributed decisively to the non-destructive behaviour.

For the entire velocity range, the present structure was proved to be less cyclic than that consisting of a circular front body. The maximum r.m.s. value of the cycle-tocycle fluctuation of the period of the structure oscillation was measured to be close to $2 \%$ up to $1.4 \mathrm{~m} \mathrm{~s}^{-1}$ and out of the transition periods.

Detailed measurements at two different velocities were performed with this structure model. The first set was performed at $0.8 \mathrm{~m} \mathrm{~s}^{-1}$ as representative of the first oscillation mode whereas the second was performed at $1.22 \mathrm{~m} \mathrm{~s}^{-1}$ to quantify the structure movement within the second. These velocity values were chosen as the best representative velocities of these modes.

Results at $0.8 \mathrm{~m} \mathrm{~s}^{-1}$

At this velocity, the Reynolds number, based on a characteristic length equal to the overall length of the model, was defined as $6.76 \times 10^{4}$. The frequency of the resulting structure movement was measured to be $2.77 \mathrm{~Hz} \pm 1.4 \%$.

Figure 13(a) shows the front body angle time-phase trace within an oscillation period. The r.m.s. value associated with the fluctuation of this quantity was measured to be $0.5^{\circ}$. In the same figure, the transverse displacement of the structure trailing edge is shown. The delay of the trailing edge movement with respect to the front body rotation was measured to be smaller than $60^{\circ}$, so the trailing edge movement could be considered to be in agreement with the rectangular front body.

In figure 13(b), the deformation of the structure for successive time-phase angles is shown. Figure 13(c) shows the successive positions of the trailing edge within an oscillation period. At the beginning of the period, the trailing edge was measured to be at the point $\left.(x ; y)\right|_{\psi=0^{\circ}}=(69.68 \mathrm{~mm} ; 10.53 \mathrm{~mm})$.

Results at $1.22 \mathrm{~m} \mathrm{~s}^{-1}$

An identical set of measurements were performed at $1.22 \mathrm{~m} \mathrm{~s}^{-1}$. At this velocity, the Reynolds number was $10.3 \times 10^{4}$ and the frequency of the resulting structure movement was measured to be $4.70 \mathrm{~Hz} \pm 0.7 \%$.

Figure 14(a) shows the time-phase trace of both the angle of the structure front body and also the transverse displacement of the trailing edge within an oscillation period. The r.m.s. value associated with the fluctuation of the front body angle was close to $0.6^{\circ}$, and the time-phase difference between the trailing edge and front body movement was measured to be approximately $65^{\circ}$, i.e. the trailing edge was delayed, moving in agreement with the front body rotation.

In figure $14(b)$, the structure deformation is presented for successive instants within an oscillation period. Figure 14(c) presents the successive positions occupied by the trailing edge within the same period. The two symmetric singular points in the plot corresponded to a complete reverse of the trailing edge movement and were registered at approximately $\psi=105^{\circ}$ and $\psi=285^{\circ}$. These values showed a good agreement between the singular points in figure $14(c)$ and the shape of the plot in figure 14(a). At the beginning of the period, the coordinates of the trailing edge were $\left.(x ; y)\right|_{\psi=0^{\circ}}=(68.39 \mathrm{~mm} ; 13.83 \mathrm{~mm})$.

The results obtained with this structure model showed that, even at higher Reynolds numbers, the structure consisting of a $4 \mathrm{~mm} \times 22 \mathrm{~mm}$ rectangular front body was not able to disturb the flow in such a way as to trigger an excitation of the IIE type at low velocities. Therefore, the results did not show oscillation modes resulting from the resonance between the vortex-shedding frequency and one of the natural frequencies of the structure. 

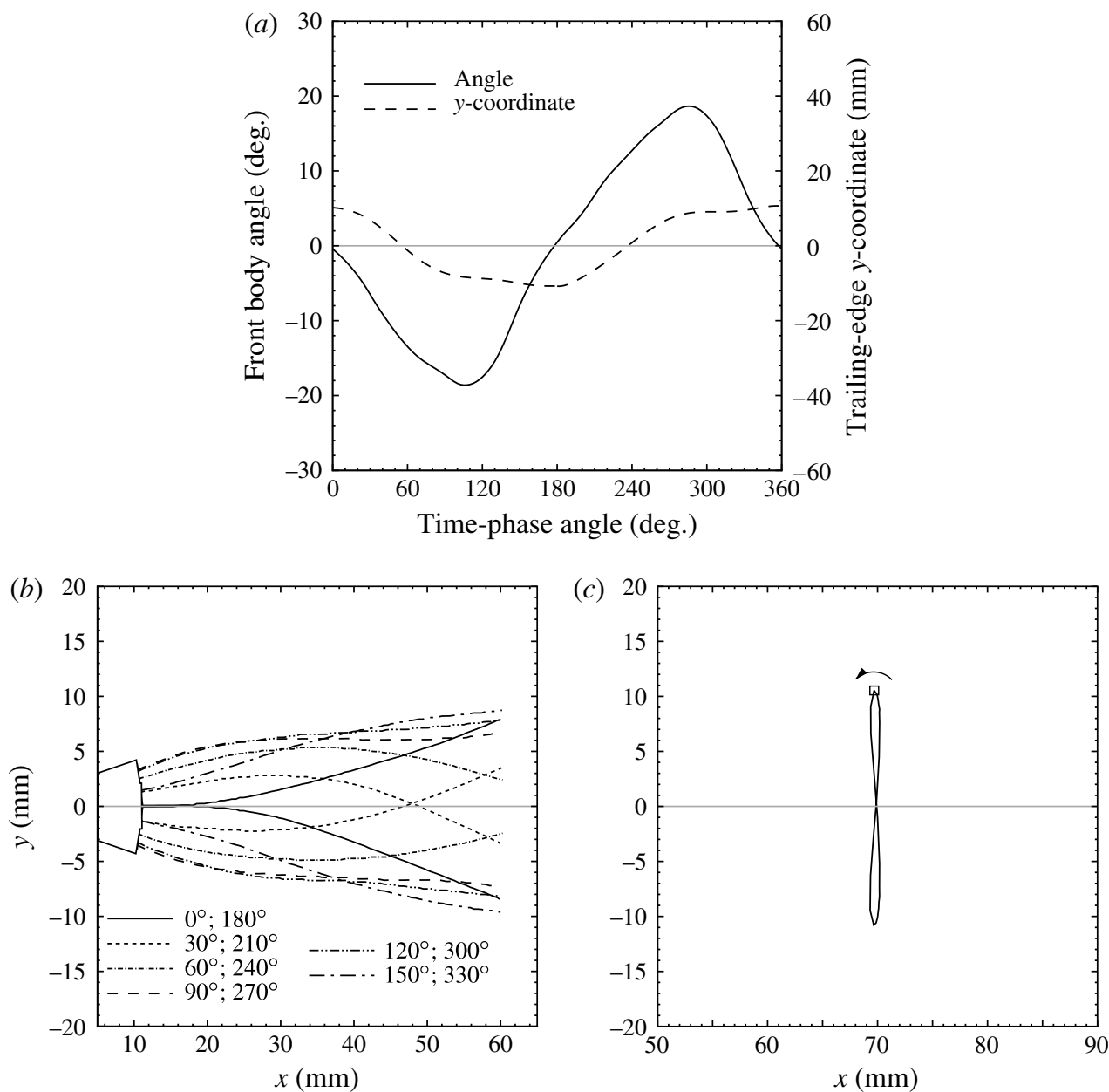

FIGURE 13. Front body angle and trailing edge $y$ coordinate $(a)$, deformation of the structure (b) and trailing edge coordinates $(c)$, within a period of motion at $0.80 \mathrm{~m} \mathrm{~s}^{-1}$ in turbulent flows, with the F2M.P4 model.

Regarding the onset of the movement, at $\sim 0.65 \mathrm{~m} \mathrm{~s}^{-1}$, the resonance condition in (1.3) gives an indication of a probable excitation of the IIE type at $\sim 0.57 \mathrm{~m} \mathrm{~s}^{-1}$, the analysis of the deformation and the high value of the first natural frequency of the structure indicate that the importance of IIE was small compared with MIE. Actually, the analysis of the deformation also indicated that the oscillation mode found between 0.65 and $0.96 \mathrm{~m} \mathrm{~s}^{-1}$ (see (a) in figure 12a) was a result of the MIE mechanism involving mode coupling between the zeroth and the first bending mode of the structure.

The MIE oscillation mode registered at higher flow velocities ((d), figure 12a) was also dominated by the zeroth and first bending modes of the structure. However, in this case, the analysis of the deformation (see figure 15) showed the presence of higher harmonics of the natural frequencies of the structure rather than just the fundamental one, as it was observed in the preceding oscillation mode. Both the shape of the 

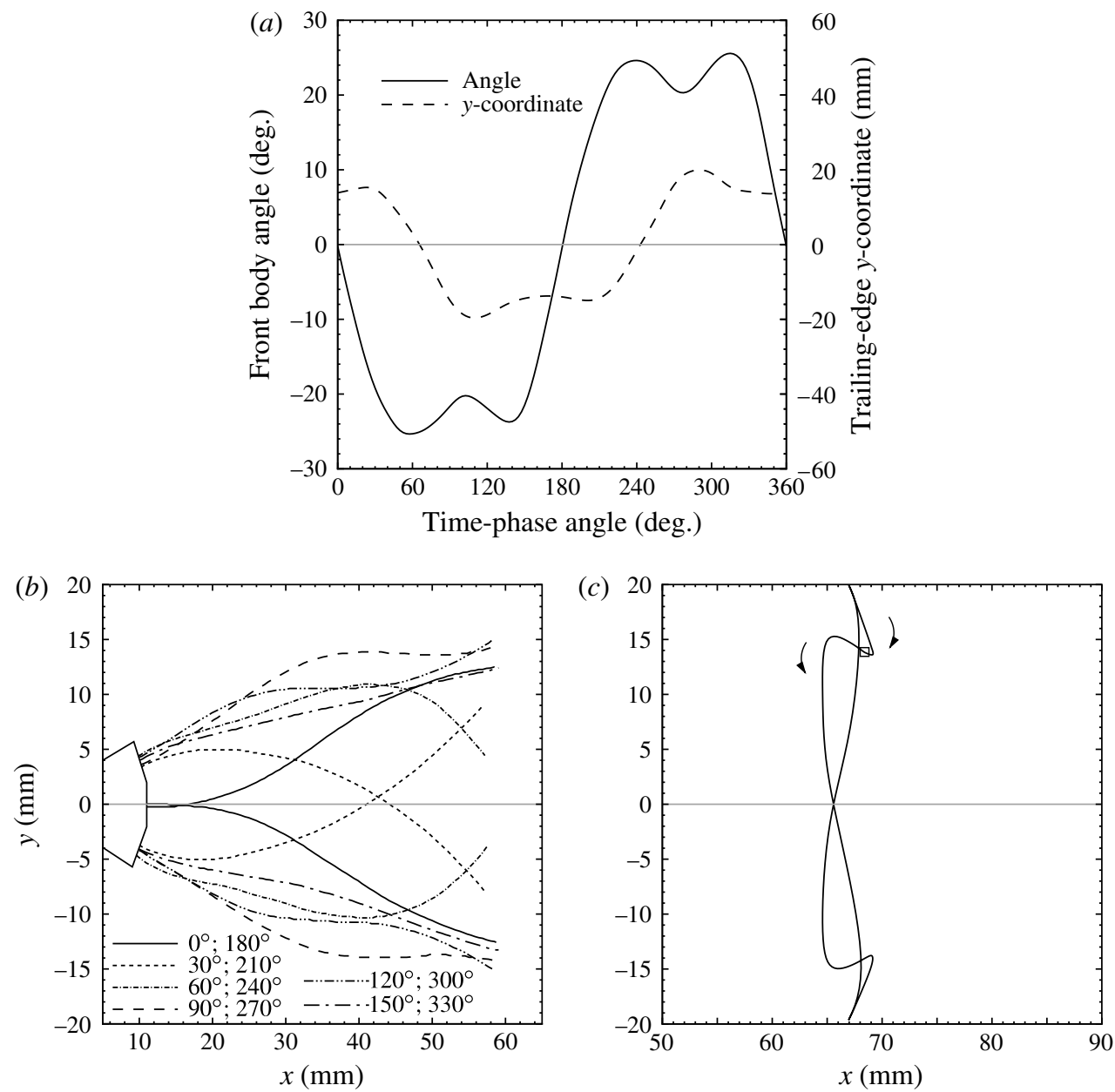

FIGURE 14. Front body angle and trailing edge $y$ coordinate $(a)$, deformation of the structure $(b)$ and trailing edge coordinates $(c)$, within a period of motion at $1.22 \mathrm{~m} \mathrm{~s}^{-1}$ in turbulent flows, with the F2M.P4 model.

bending mode coefficients time-phase traces and the lower level of energy contained in the first and higher bending modes support this conclusion.

Figure 15 also explains the unique deformation measured at $1.22 \mathrm{~m} \mathrm{~s}^{-1}$ (see figure $14 a-c$ ). This type of movement was never observed for the structures consisting of a circular front body and was different to the movement exhibited by the same structure at similar velocities in laminar flows. One may observe that, although unusual, the results of the structure deformation corresponding to this oscillation mode are conclusive. Hence, in figure 14(c), the loops outside the classical 'figure-of-eight' shape correspond exactly to the regions between the two local positive and negative maxima in the curve in figure 14(a). In addition, the points of reverse in the trailing edge trajectory match the local positive and negative minimum registered in the timephase trace of the front body angle of the structure $\left(\psi \approx 105^{\circ}\right.$ and $\psi \approx 285^{\circ}$, see figure $14 a$ ). 

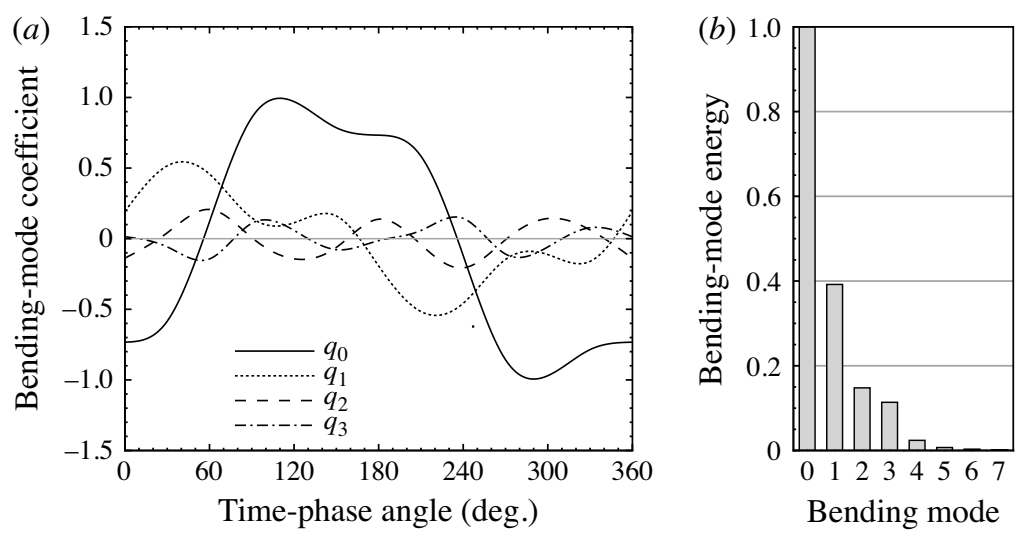

FIGURE 15. Decomposition of the F2M.P4 model deformation in the individual bending modes at $1.22 \mathrm{~m} \mathrm{~s}^{-1}$ in turbulent flows.
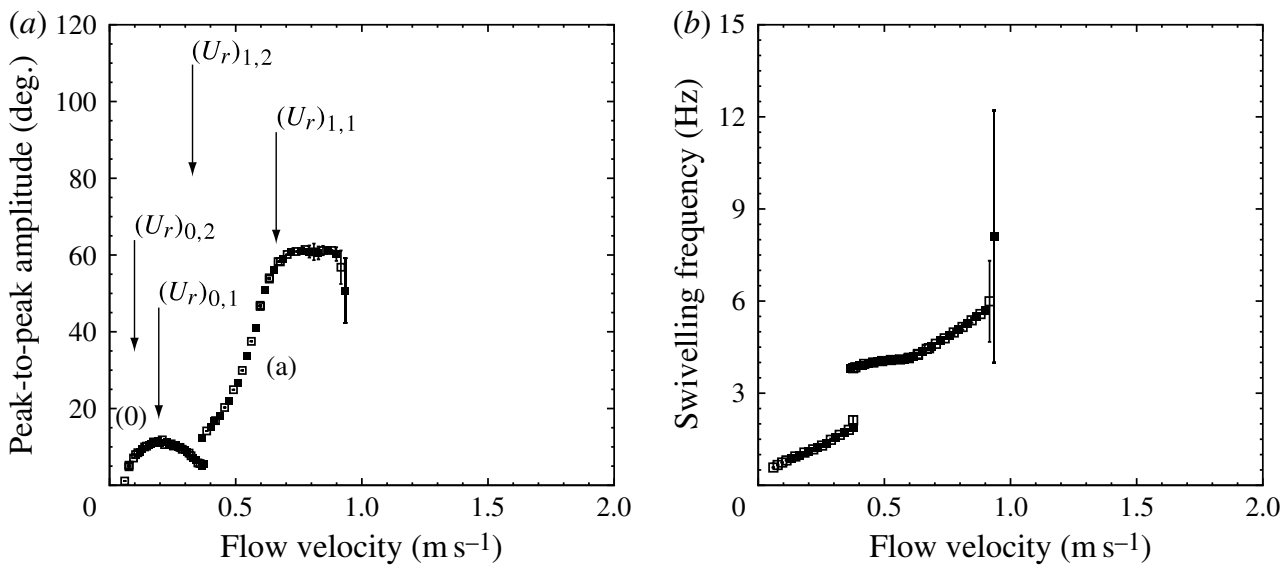

FIGURE 16. Oscillation amplitude $(a)$ and frequency $(b)$ versus flow velocity for the F2M.CH model in turbulent flows.

\subsubsection{Results with the F2M.CH model}

Figure 16 shows the elastic-dynamic response of the structure consisting of a circular front body as a function of the approaching flow velocity (arrows indicate the critical velocity values according to (1.1)).

In water flows, this structure could be excited to a periodic LCO movement at very low velocities. The onset of the movement was registered for a flow velocity slightly higher than $0.1 \mathrm{~m} \mathrm{~s}^{-1}$. Visualizations showed that the resulting oscillation mode (see (0) in figure 16a) was associated with the rigid body motion of the model, i.e. the structure swivelled in the fluid around its free rotating axle without changing its original, straight shape. Because it corresponded to rigid body motion, this mode was named the zeroth oscillation mode. As an example of this mode, figure 17 shows the time-phase trace of the front body angle and the deflection of the structure for successive time-phase angles at $0.19 \mathrm{~m} \mathrm{~s}^{-1}$. 

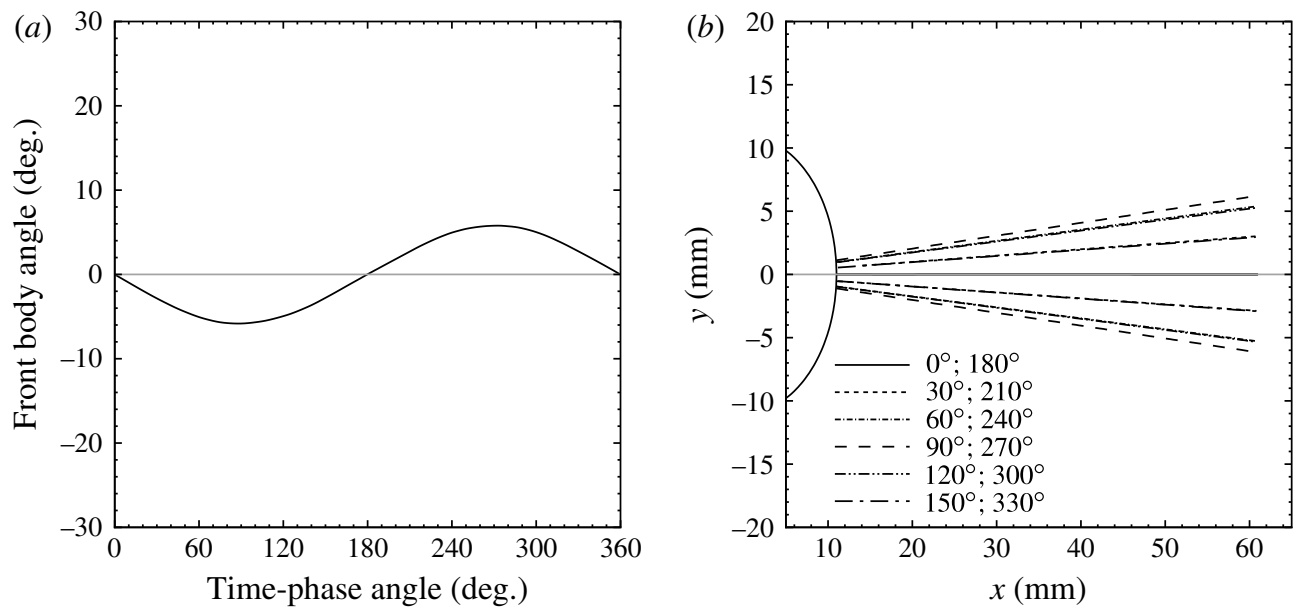

FIGURE 17. Front body angle $(a)$ and deformation of the structure $(b)$ within a period of motion at $0.19 \mathrm{~m} \mathrm{~s}^{-1}$ in turbulent flows, with the F2M.CH model.

An oscillation-mode transition was observed for a flow velocity close to $0.4 \mathrm{~m} \mathrm{~s}^{-1}$ and it was characterized by an abrupt change of both the amplitude and frequency of the structure movement. In the new oscillation mode (see (a) in figure 16a), the behaviour of the structure was in all respects similar to the first oscillation mode exhibited by the same model in laminar flows; the structure deformation was dominated by the first bending mode of the structure and the movement of the trailing edge was in agreement with the rotation of the front body.

On further increasing the approaching flow velocity, an unusual behaviour was observed. As soon as the amplitude of the structure started to decrease, after reaching a local maximum, the motion characteristics degraded very rapidly. This behaviour was supported by the error bars in figure 16. The coupled movement became nonperiodic and non-symmetric and led to rapid destruction of the structure. Therefore, no measurements could be obtained for flow velocities higher than $0.9 \mathrm{~m} \mathrm{~s}^{-1}$. This sequence of events occurred when the oscillation frequency was showing the first signals of transition to a new oscillation mode.

Both oscillation-mode transitions occurred when the frequency of the structure movement approached one of the natural frequencies of the structure. At $0.37 \mathrm{~m} \mathrm{~s}^{-1}$, the structure oscillation frequency was approximately $f_{0}=1.9 \mathrm{~Hz}$ and at $0.9 \mathrm{~m} \mathrm{~s}^{-1}$ approximately $f_{1}=5.89 \mathrm{~Hz}$. In the range in which the structure movement was periodic and reproducible, up to $0.9 \mathrm{~m} \mathrm{~s}^{-1}$, the r.m.s. value associated with the cycleto-cycle fluctuation of the motion period was measured to be lower than $1 \%$.

Apart from the trivial rigid body oscillation mode, the only oscillation mode that could be characterized in detail using the present structure was the first one. Therefore, detailed measurements were performed in water just at $0.68 \mathrm{~m} \mathrm{~s}^{-1}$. This value was selected because it was located close to the velocity of maximum structure amplitude excitation (see figure $16 a$ ).

Results at $0.68 \mathrm{~m} \mathrm{~s}^{-1}$

At this velocity, the Reynolds number, based on the length of the structure, was close to $5.75 \times 10^{4}$ and the frequency of the structure movement was measured to be 

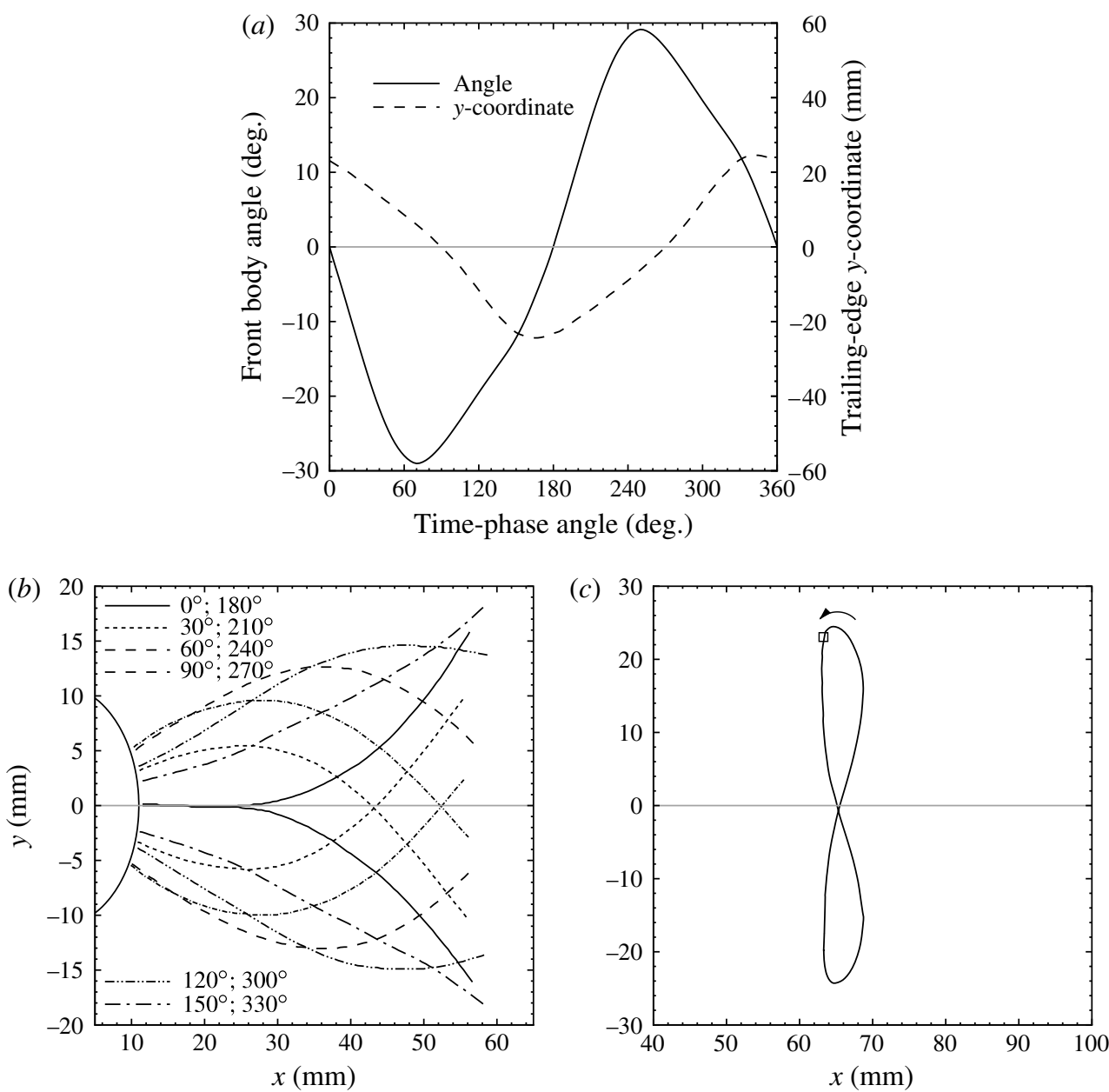

FIGURE 18. Front body angle and trailing edge $y$ coordinate $(a)$, deformation of the structure $(b)$ and trailing edge coordinates $(c)$, within a period of motion at $0.68 \mathrm{~m} \mathrm{~s}^{-1}$ in turbulent flows, with the F2M.CH model.

$4.45 \mathrm{~Hz} \pm 0.4 \%$. The Reynolds number, based on the diameter of the structure circular front body, was approximately $1.54 \times 10^{4}$.

Figure 18(a) shows the time-phase traces of the angle of the front body and transverse displacement of the structure trailing edge within an oscillation period. The delay between the movement of the trailing edge and the front body was measured to be approximately $95^{\circ}$ and the r.m.s. value associated with the fluctuation of the front body angle was measured to be smaller than $0.6^{\circ}$.

Figure $18(b, c)$ show successive time-phase resolved deformations of the structure and the trajectory followed by the trailing edge during an oscillation period. At the beginning of the period, the trailing edge was measured to be at the point $\left.(x ; y)\right|_{\psi=0^{\circ}}=(63.26 \mathrm{~mm} ; 23.10 \mathrm{~mm})$.

In laminar flows, the excitation of the movement of this structure was only possible for flow velocities higher than $0.35 \mathrm{~m} \mathrm{~s}^{-1}$ because of the restriction imposed by the critical Reynolds number for the formation of a regular vortex shedding past a circular 
cylinder $(R e \geqslant 47)$. Because of the increase in the Reynolds number, this limitation disappeared in turbulent flows and an oscillation mode associated with the zeroth oscillation mode of the structure (see (0) in figure 16a) found favourable conditions to occur at very low flow velocities, before the excitation of the first bending mode.

Because the amplitude of the structure movement remained small within the entire zeroth mode, a significant alteration of the characteristics of the flow fluctuation past the structure as a consequence of its movement was not observed. Consequently, this was the only pure IIE oscillation mode registered during the present study. In support of this conclusion, just the zeroth bending mode of the structure could be identified in the deformation of the structure presented in figure 17.

In many other cases (as in the first oscillation mode exhibited by this structure model), the IIE mechanism of excitation results in a large increase of the resulting movement amplitude. When the amplitude increases beyond a certain limit, the movement of the oscillating structure deforms further the flow and alters the characteristics of the flow fluctuation past the structure. If the perturbations in the flow field stimulate the transfer of fluid energy between the bending mode excited by the IIE and any other bending mode, or modes, of the structure, the excitation of the IIE type is followed by an MIE excitation involving mode coupling. It can also occur that the perturbations introduced in the flow by the movement of the structure are sufficiently large to change the fluid forces in such a way that they produce positive work on the structure and energize the same bending mode originally excited by the IIE mechanism. In this case, IIE is followed by MIE independent of coupling. In the case of the first oscillation mode of the present test case (see (a) in figure 16a), the self-sustained MIE mechanism of transfer of energy between the first and zeroth bending mode was triggered after the excitation of the first bending mode of the structure through the IIE mechanism.

The results obtained in the turbulent regime also highlighted the importance of the higher harmonic components of the natural frequencies of the structure in the IIE excitation mechanism. Because of the low fluid damping, the excitation of the oscillation modes was observed not only at flow velocities at which the flow fluctuation was in resonance with the fundamental value of the natural frequencies of the structure (as was found in laminar flows) but also when the flow was in resonance with higher harmonic components of the natural frequencies. This observation is clearly supported by figure $16(a)$. At $\sim 0.1 \mathrm{~m} \mathrm{~s}^{-1}$, equation (1.1) shows a close relation between the Strouhal number and the second harmonic of the zeroth natural frequency of the structure $\left(f_{0} / 2=0.95 \mathrm{~Hz}\right)$. This fact supports the statement that at the onset of the movement, the vortex shedding past the front body was in resonance with the zeroth natural frequency of the structure. After the initial excitation and on increasing the flow velocity, a second resonance was found within the same oscillation mode (see (0) in figure 16a) at $\sim 0.2 \mathrm{~m} \mathrm{~s}^{-1}$ involving the fundamental value of the zeroth natural frequency $\left(f_{0}=1.9 \mathrm{~Hz}\right)$. This resonance coincided with a point of local maximum excitation of the structure.

The same equation shows a direct relation between the movement excitation and the flow fluctuation for the first oscillation mode, at $\sim 0.33 \mathrm{~m} \mathrm{~s}^{-1}$, on comparing the Strouhal number and the second harmonic component of the first natural frequency $\left(f_{1} / 2=2.95 \mathrm{~Hz}\right.$ ). As in the previous case, this oscillation mode (see (a) in figure 16a) was characterized by a local maximum at the same point where the flow was in resonance with the fundamental value of the first natural frequency of the structure $\left(f_{1}=5.89 \mathrm{~Hz}\right)$. Figure 19 shows the results of the analysis of the deformation at $0.68 \mathrm{~ms}^{-1}$. 

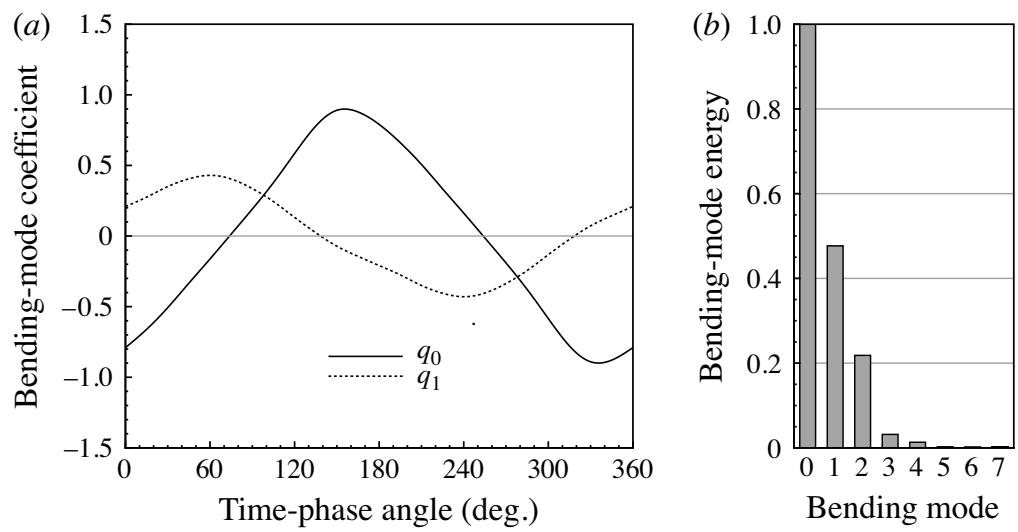

FIGURE 19. Decomposition of the F2M.CH model deformation in the individual bending modes at $0.68 \mathrm{~m} \mathrm{~s}^{-1}$ in turbulent flows.
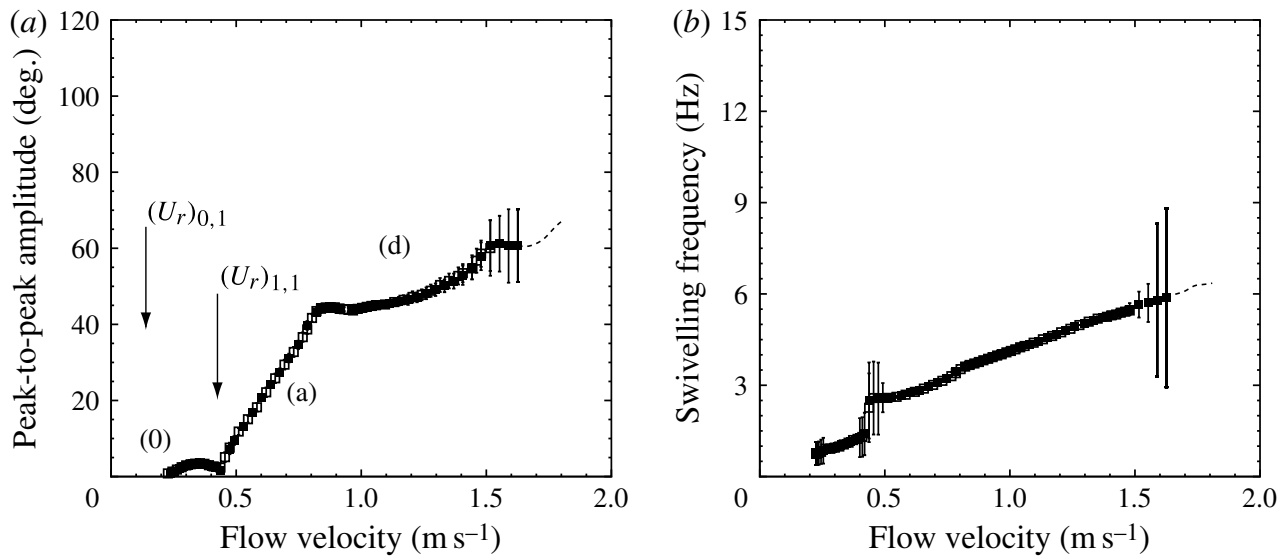

FIGURE 20. Oscillation amplitude $(a)$ and frequency $(b)$ versus flow velocity for the F2M.P8 model in turbulent flows.

As far as the frequency is concerned, figure 16(b) shows that the oscillation frequency increased linearly with the velocity of the approaching flow. The only exception to this occurred at the beginning of the first oscillation mode, between 0.37 and $0.6 \mathrm{~m} \mathrm{~s}^{-1}$, where a very pronounced 'locking-in' region was registered.

\subsubsection{Results with the F2M.P8 model}

This structure was similar to the F2M.P4 model but with a thicker rectangular front body. Its elastic-dynamic response is presented in figure 20 as a function of the flow (arrows indicate the critical velocity values according to (1.3)).

The first analysis of both plots immediately revealed that the elastic-dynamic response of this structure was more difficult to interpret than any other investigated up to this point. For the first time, it was not possible to identify clearly the different oscillation modes which controlled the movement of the structure from a simple analysis of the oscillation frequency (see figure 20b). Instead, the analysis of the 
modes could only be concluded by correlating the results from figures $20(a)$ with $20(b)$ and resulted in the identification of three distinct oscillation modes.

The initial oscillation mode (see (0) in figure 20a) could be excited for flow velocities of $\sim 0.2 \mathrm{~m} \mathrm{~s}^{-1}$ and it was mostly characterized by a tenuous oscillation of the structure; the maximum excitation was observed at $3.5 \mathrm{~m} \mathrm{~s}^{-1}$ at the same time the deflection of the front plate was limited to $\pm 2.1^{\circ}$. Although this oscillation mode could not be considered as a pure rigid body movement, visualizations proved that the deformation of the structure was minimal.

Around $0.43 \mathrm{~m} \mathrm{~s}^{-1}$, a transition to a new oscillation mode was observed. It occurred when the oscillation frequency was close to the structure zeroth natural frequency $\left(f_{0}=1.9 \mathrm{~Hz}\right)$ and it was characterized by a significant fluctuation of the movement frequency. The last observation is supported by the error bars in figure $20(b)$. No fluctuations were registered in the movement amplitude results. During the excitation of this oscillation mode (see (a) in figure 20a), the elastic-dynamic response of the structure proved to be as simple as possible. The amplitude of the movement varied linearly with the flow velocity and the deformation of the structure was exclusively dominated by the first bending mode of the structure. In connection with this, the movement of the trailing edge was observed to be in agreement with the rotation of the rectangular front body.

At $\sim 0.8 \mathrm{~m} \mathrm{~s}^{-1}$, a significant change in the curve in figure $20(a)$ was registered, indicating that a new self-exciting mechanism was competing for the control of the structure movement and forcing an oscillation-mode transition. This transition was also perceptible in figure $20(b)$ by a slight variation in the slope of the frequency plot. The new oscillation mode (see (d) in figure 20a) was in all respects similar to that registered with the thinner rectangular front body structure and resulted in the same 'figure-of-M'-shaped front body time-phase trace and unusually deformed trailing edge trajectory. For this oscillation mode, the movement of the trailing edge could be considered to be in agreement with the rotation of the front body.

On further increasing the velocity, the structure movement became non-symmetric and non-periodic and lead repeatedly to the destruction of the model close to $1.8 \mathrm{~m} \mathrm{~s}^{-1}$. This rapid succession of events occurred at the same time that the oscillation frequency was close to the first natural frequency of the structure $\left(f_{1}=5.6 \mathrm{~Hz}\right)$.

For the entire velocity range of the tests, the present structure was proved to be less cyclic than that consisting of a circular front body. Within the lowest oscillation mode, up to $0.43 \mathrm{~m} \mathrm{~s}^{-1}$, the r.m.s. value associated with the cycle-to-cycle fluctuation of the period of the movement reached $3 \%$. Within the other oscillation modes, up to $1.5 \mathrm{~m} \mathrm{~s}^{-1}$, the r.m.s. value decreased to half, i.e. it was limited to $1.5 \%$. Close to $1.5 \mathrm{~m} \mathrm{~s}^{-1}$, the r.m.s. values associated with the fluctuation of both movement frequency and amplitude was observed to increase.

For the present structure, detailed measurements were performed for an approaching flow velocity of 0.8 and $1.22 \mathrm{~m} \mathrm{~s}^{-1}$. They were chosen as the best representatives of both oscillation modes and to match the velocity values investigated with the thinner rectangular front body structure.

\section{Results at $0.68 \mathrm{~m} \mathrm{~s}^{-1}$}

At this flow velocity, the Reynolds number, based on the overall length of the structure model, was $5.75 \times 10^{4}$ and the frequency of the structure oscillation was measured to be $2.87 \mathrm{~Hz} \pm 1 \%$. 

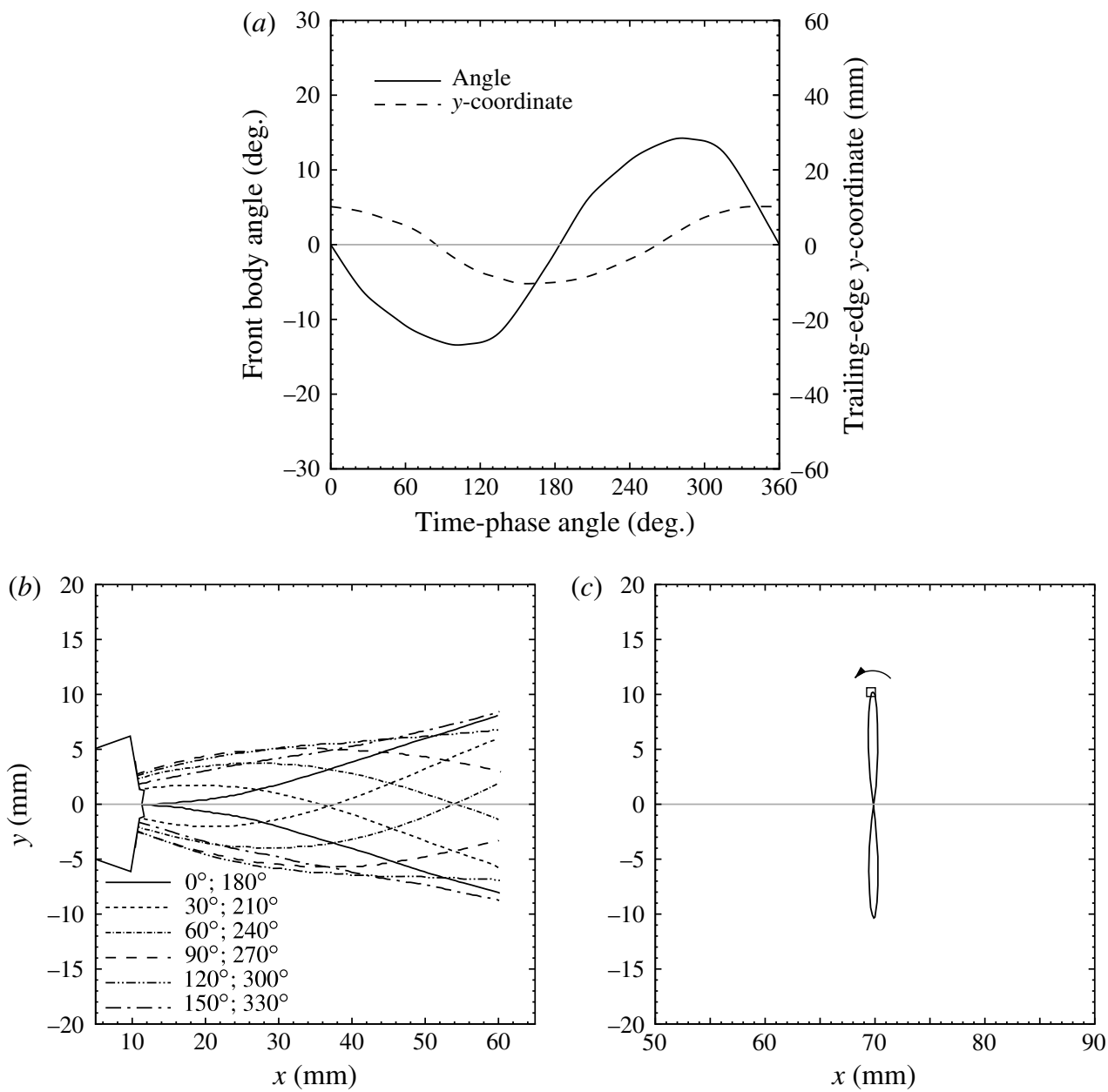

FIGURE 21. Front body angle and trailing edge $y$ coordinate $(a)$, deformation of the structure $(b)$ and trailing edge coordinates $(c)$, within a period of motion at $0.68 \mathrm{~m} \mathrm{~s}^{-1}$ in turbulent flows, with the F2M.P8 model.

Figure 21(a) shows the time-phase trace of the rectangular front body angle and the transverse displacement of the trailing edge within an oscillation period. The two curves in figure 21(a) indicate that the movement of the trailing edge was in agreement with the rotation of the front body. The delay was measured to be approximately $75^{\circ}$. The r.m.s. value associated with the fluctuation of the angle was $0.6^{\circ}$.

Figure 21(b) shows the deformation of the structure for successive time-phase angles. In figure 21(c), the trajectory followed by the trailing edge within an oscillation period is shown. At the beginning of the oscillation period, the trailing edge was measured to be at the point $\left.(x ; y)\right|_{\psi=0^{\circ}}=(69.70 \mathrm{~mm} ; 10.17 \mathrm{~mm})$.

Results at $1.22 \mathrm{~m} \mathrm{~s}^{-1}$

A similar set of measurements were performed at $1.22 \mathrm{~m} \mathrm{~s}^{-1}$, which corresponded to a Reynolds number of $10.3 \times 10^{4}$. At this velocity, the frequency of the structure movement was measured to be $4.71 \mathrm{~Hz} \pm 1 \%$. 

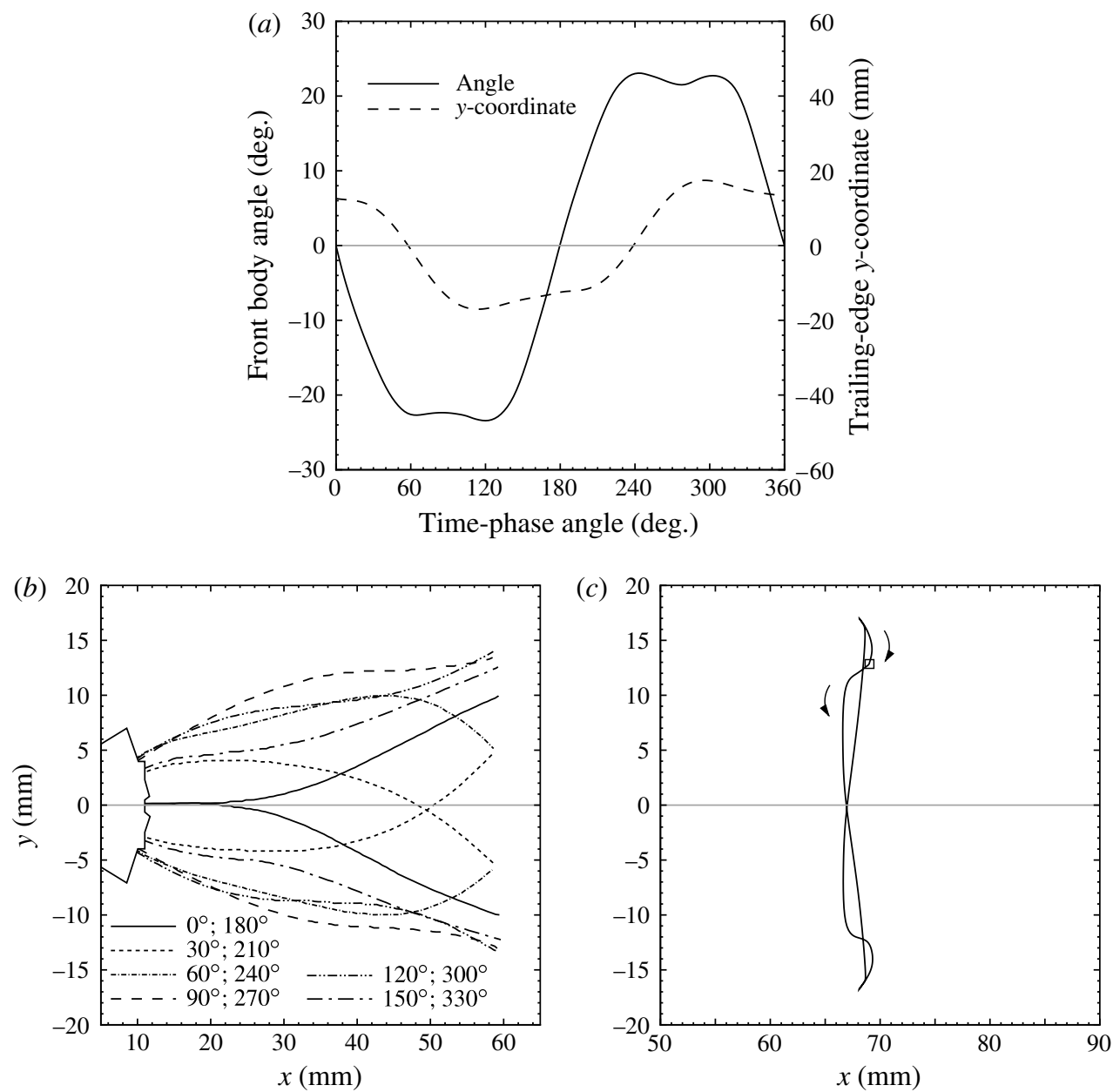

FIGURE 22. Front body angle and trailing edge $y$ coordinate $(a)$, deformation of the structure $(b)$ and trailing edge coordinates $(c)$, within a period of motion at $1.22 \mathrm{~m} \mathrm{~s}^{-1}$ in turbulent flows, with the F2M.P8 model.

Figure 22(a) presents the time-phase trace of the front body angle and the transverse displacement of the structure trailing edge within an oscillation period. The r.m.s. value associated with the fluctuation of the angle was $0.6^{\circ}$. The curves in figure 22(a) show that the trailing edge was still moving in agreement with the rotation of the front body, with a time-phase delay of $\sim 60^{\circ}$.

Figure $22(b, c)$ presents the structure deformation for successive instants, and the trajectory followed by the trailing edge within an oscillation period. The latter exhibited the same unusual shape as already observed with the structure consisting of a thinner rectangular front body at the same flow conditions. In this case, the singular points were captured at approximately $\psi=120^{\circ}$ and $\psi=300^{\circ}$. The trailing edge position at the beginning of the period was measured to be $\left.(x ; y)\right|_{\psi=0^{\circ}}=(68.98 \mathrm{~mm} ; 12.80 \mathrm{~mm})$.

The results obtained with this structure were of special interest as its elastic-dynamic response combined characteristics from the models of the previous 

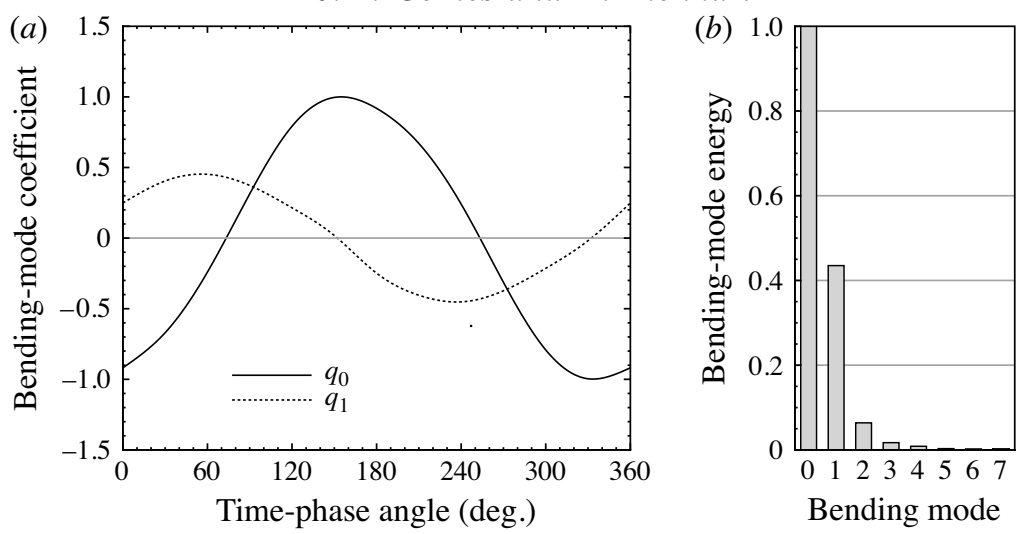

FIGURE 23. Decomposition of the F2M.P8 model deformation in the individual bending modes at $0.68 \mathrm{~m} \mathrm{~s}^{-1}$ in turbulent flows.

structures. Like the structure model consisting of a circular front body, this structure considered a front body with the characteristics of a bluff body but rectangular. Consequently, the onset of the movement of this structure was registered at very low flow velocities and the two oscillation modes exhibited at lower velocities (see (0) and (a) in figure 20a) were associated with an excitation of the IIE type. One may remember that, because of the high Reynolds number, the IIE excitation of this structure was not restricted by the critical Reynolds number for the formation of a regular vortex shedding past the front cylinder.

In a similar way as for all of the other IIE oscillation modes, a direct relation between the movement excitation and the vortex shedding past the structure front body was proved to exist. For the zeroth $\left(f_{0}=1.90 \mathrm{~Hz}\right)$ and first structure natural frequencies $\left(f_{1}=5.61 \mathrm{~Hz}\right)$, the resonance condition expressed by $(1.3)$, based on the dimensions of the rectangular front body, was satisfied at $\sim 0.138$ and $0.41 \mathrm{~m} \mathrm{~s}^{-1}$, respectively. These critical values are identified in figure 20(a) and are in good agreement with the onset of the movement of the structure and transition to the first oscillation mode, respectively.

In sum, the two IIE oscillation modes exhibited by the this model at flow velocities up to approximately $0.8 \mathrm{~m} \mathrm{~s}^{-1}$ had very similar characteristics as the oscillation modes exhibited by the circular front body structure model within the same flow velocity range. The IIE oscillation mode associated with the zeroth bending mode of the structure could be considered as almost pure. The resulting movement of the structure within this oscillation mode was very similar to that presented in figure 17. A comparison of figures 19 and 23 is conclusive in showing that the IIE oscillation mode associated with the first bending mode of the two structures were also very similar.

In opposition with what was observed at lower velocities, the oscillation mode found for velocities higher than $0.8 \mathrm{~m} \mathrm{~s}^{-1}$ was excited by the MIE mechanism and it was similar to that exhibited by the thinner rectangular front body structure for velocities higher than $0.96 \mathrm{~m} \mathrm{~s}^{-1}$. This observation is supported by the fact that the curves in figures $12(b)$ and $20(b)$ are coincident. Simultaneously, the analysis of the deformation of both structures at $1.22 \mathrm{~m} \mathrm{~s}^{-1}$ are in good agreement (see figures 15 and 24). In both figures, the time-phase shift between the zeroth and the first bending mode was approximately $69.4 \mathrm{~m} \mathrm{~s}^{-1}$. 

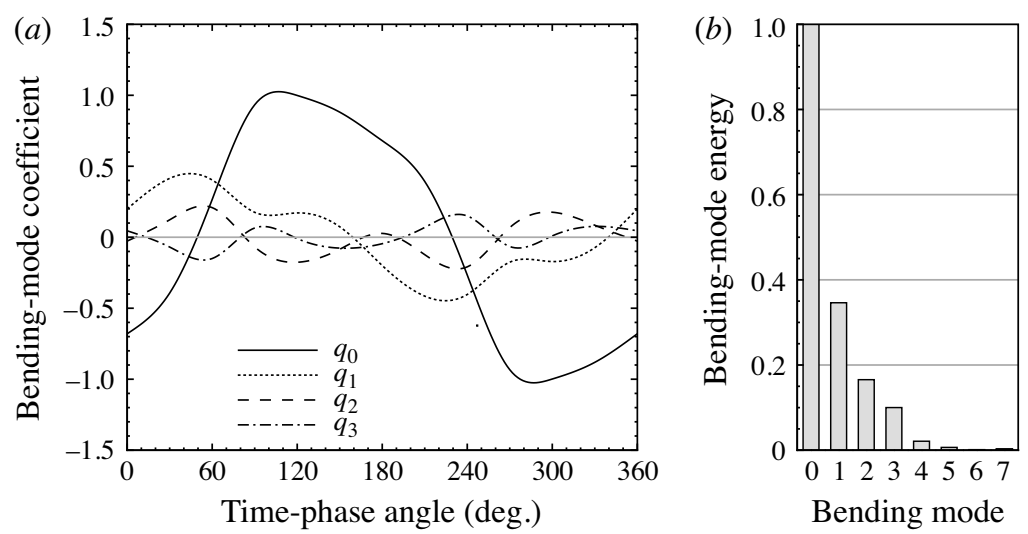

FIGURE 24. Decomposition of the F2M.P8 model deformation in the individual bending modes at $1.22 \mathrm{~m} \mathrm{~s}^{-1}$ in turbulent flows.

\section{Conclusions}

The results presented have shown that, for all structures, a symmetric and reproducible two-dimensional LCO movement could be easily self-excited for flow velocities up to $2 \mathrm{~m} \mathrm{~s}^{-1}$ within a very short time (a few oscillation cycles). After the onset of the structures movement, and on increasing the flow velocity, the FSI test cases were characterized by a sequence of clearly defined IIE and MIE excitations of the coupled fluid and structure motion. Both self-excitation mechanisms proved to be reproducible and were characterized by a unique set of properties.

Within all velocities tested, the resulting movement of the structures proved to be very symmetric. The r.m.s. value associated with the cycle-to-cycle fluctuation of the front body angle remained approximately constant at $0.5^{\circ}$ for all tests. The maximum structure deformation asymmetry was measured as \pm 0.76 and $\pm 0.58 \mathrm{~mm}$ in the tests in the laminar and turbulent regime, respectively. As far as the periodicity of the movement is concerned, the cycle-to-cycle fluctuation of the structure oscillation frequency were observed to be higher in turbulent than in laminar flows. While the maximum r.m.s. value associated with this quantity was measured as $0.9 \%$ in laminar flows, it was measured close to $2.8 \%$ in turbulent flows. This fact was a direct consequence of the lower damping added by the water to the oscillating system. For the same reason, the frequency of the resulting LCO movement was also considerably higher.

At lower velocities, the movement was mostly controlled by the IIE mechanism. Excitations of this kind were observed the vortex shedding past the structure front body was in resonance with one of the fundamental natural frequencies, or harmonic components, of the structure.

Thus, in laminar flows, the onset of the movement of the structure consisting of a circular front body, at $\sim 0.75 \mathrm{~m} \mathrm{~s}^{-1}$, was observed at the same time that the vortexshedding frequency was close to the first natural frequency of the structure. At this velocity, the evidences of a 'locking-in' region were found to be almost imperceptible. This fact was a direct consequence of the high damping added by the surrounding liquid to the oscillation system.

For the same flow conditions, no excitations of the IIE type were found with the structure consisting of a rectangular front body. This difference in behaviour 
emphasized the direct relation between the IIE excitation mechanism at low Reynolds number and the beginning of the laminar regular vortex shedding past the front body of the structure. The analysis of the results obtained with the structure consisting of a $8 \mathrm{~mm} \times 22 \mathrm{~mm}$ rectangular front body revealed that the velocity needed to achieve critical Reynolds number for the onset of the laminar vortex-shedding regime $(R e \approx 47)$ was far higher that the resonance velocity associated with the zeroth bending mode of the structure. For that reason, the IIE excitation of the structure movement associated with its zeroth bending mode was not observed. Because the velocity needed to achieve the critical Reynolds number was significantly higher for the structures consisting of a more slender shape, IIE excitations associated with both the zeroth and also the first bending modes were not registered for the structure consisting of a $4 \mathrm{~mm} \times 22 \mathrm{~mm}$ rectangular front body.

This limitation disappeared in turbulent flows because of the increase in the Reynolds number, and the flow around the front body of the structures was characterized by a vortex shedding, starting from flow velocities very close to zero. Therefore, the onset of the movement of the structures was observed at very low flow velocities as a consequence of an IIE excitation involving the resonance of the flow fluctuation and the zeroth bending mode of the structure. This excitation was followed by a transition to a new oscillation mode in which the fluctuation of the flow was in resonance with the first bending mode, and was observed for the structures consisting of a circular and a thick rectangular front body. In both case, the structures exhibited a clear 'locking-in' region at the transition to the first oscillation mode in which the vortex shedding frequency was locked to the oscillation frequency of the structure, and vice-versa. This observation confirmed that 'locking-in' regions are more pronounced, and therefore easier to observe, in turbulent flows because of the much lower added damping introduced by the fluid.

In addition, the results showed that IIE resonance processes at higher Reynolds numbers can be induced not only at the fundamental value of the natural frequencies of the structure but also at the harmonics of these values. Once again, this fact was associated with the reduction of the added damping introduced by the fluid into the oscillation system as a consequence of the reduction of the fluid viscosity. Regarding the movement excitation of the structure consisting of the slender rectangular front body, it was demonstrated that it was too thin to trigger any excitation of the IIE type.

Finally, because the second natural frequency of the structures was always bigger that the shedding frequencies registered during both laminar and turbulent tests, no IIE oscillation modes associated with the second, or higher, bending modes were observed.

All of the results were conclusive in showing that excitations of the IIE type were uniquely determined by the geometry of the structure front body. The afterbody geometry or the material of the structure proved to have no significant influence on the IIE mechanism except for the effects on the dynamic characteristics of the model, such as the moment of inertia and natural frequencies. As far as the properties of the fluid are concerned, the influence of the approaching flow velocity in the IIE mechanism was very strong as it directly influenced the characteristics of the vortex shedding past the structure front body. In contrast, because the Strouhal number does not change considerably with the Reynolds number in the range of the tests, the IIE mechanism was not influenced by the Reynolds number and the resulting IIE oscillation modes had similar responses in both flow regimes.

At higher velocities, the amplitudes of the flow disturbances created by the movement of the structure increased and excitations of the MIE type found favourable conditions to be triggered. For all investigated test cases, the MIE proved to be 
the dominant self-excitation mechanism for flow velocities above $1 \mathrm{~m} \mathrm{~s}^{-1}$, both in turbulent and laminar flows.

The modal analysis of the structures deformation at different flow velocities showed that structure bending modes up to third order were significantly involved in the deformation of the structures and consequently in the MIE excitation processes. In total, four different MIE swivelling modes involving mode coupling were identified, two in laminar and two in turbulent flows. All of them were characterized by exchange of energy between a single exciting bending mode and the rest of the significant bending modes involved in the deformation of the structure. In the first MIE swivelling mode found in laminar flows (see (b) in figures 3 and 7), the second bending mode was the exciting mode. In the second swivelling mode (see (c) in figure 3), the exciting mode was the third.

In turbulent flows, two MIE swivelling modes were found to be associated with the first bending mode of the structure. In the first swivelling mode (see (a) in figure 12), the bending mode associated with the fundamental value of the first natural frequency was the excited mode and exchanged energy with the zeroth and second bending modes of the structure. In the other (see (d) in figures 12 and 20), the same mechanism was observed but involving higher harmonics of the bending modes of the structure.

Regarding the interaction between the bending modes involved in the structure deformation, the measured time-phase shift between the exciting bending mode and the others varied between approximately 70 and $110^{\circ}$. On comparing the exciting bending mode with the zeroth bending mode alone, which proved to be the most important bending mode of the structure in the energy transfer processes, the average value of the time-phase shift was $97^{\circ}$. This value was in fairly good agreement with the theoretical value of $90^{\circ}$, without which a self-sustained exchange of energy between bending modes of the structure would be impossible.

Further conclusions regarding the MIE mechanism of excitation could be drawn by comparing the MIE oscillation modes exhibited by different structures at similar flow conditions. In turbulent flows, this happened for example for the two structures consisting of a rectangular front body at flow velocities higher than approximately $0.9 \mathrm{~m} \mathrm{~s}^{-1}$. Within this range of velocities, the elastic-dynamic response of the structures and the resulting oscillation modes (see (d) in figures 12 and 20) were identical. In particular, curves in figures $12(b)$ and $20(b)$ coincided. The same conclusion was achieved on comparing the MIE oscillation mode exhibited by the structures consisting of a rectangular and a circular front body in laminar flows (see (b) in figures 3 and 7).

These results were conclusive in showing that self-excitations of the MIE type were not influenced by the shape of the structure. Instead, this mechanism was sensitive to the dynamic characteristics of the structures and, in particular, to the properties of the fluid. The Reynolds number proved to be the most significant parameter in the MIE excitation and its effects were highlighted on comparing, for example, the elastic-dynamic response of the structure consisting of a circular front body in turbulent and laminar flows. Whereas the MIE excitations involved mainly the lower bending modes of the structure in turbulent flows (specifically the zeroth and first bending modes), in laminar flows they involved bending modes up to third order. 


\section{Acknowledgements}

The authors gratefully acknowledge the financial support for their research work through the German Science Foundation (DFG), Germany, and Fundação para a Ciência e a Tecnologia (FCT), Portugal. In addition the authors acknowledge the funding of the Erlangen Graduate School in Advanced Optical Technologies (SAOT) by DFG in the framework of the German excellence initiative.

\section{REFERENCES}

Fey, C., Konig, M. \& Eckelmann, H. 1998 A new Strouhal-Reynolds-number relationship for the circular cylinder in the range $47<R e<2 \times 10^{5}$. Phys. Fluids 10 (7), 1547-1549.

Gomes, J. P. \& Lienhart, H. $2010 a$ Experimental benchmark: self-excited fluid-structure interaction test cases. In Fluid Structure Interaction II (ed. H. J. Bungartz, M. Mehl \& M. Schäfer), Lecture Notes in Computational Science and Engineering, Vol. 73, pp. 383-411. Springer.

Gomes, J. P. \& Lienhart, H. $2010 b$ Time-phase resolved optical measurements on twodimensional FSI problems. Phys. Procedia 5, 679-688.

Gomes, J. P., Yigit, S., Lienhart, H. \& SChëfer, M. 2010 Experimental and numerical study on a laminar fluid-structure interaction reference test case. J. Fluids Struct. 27 (1), 43-61.

Komatsu, S. \& Kobayashi, H. 1980 Vortex-induced oscillation of bluff cylinders. J. Wind Engng Ind. Aerodyn. 6, 335-362.

KNISELY, C. W. 1990 Strouhal numbers of rectangular cylinders at incidence: a review and new data. J. Fluids Struct. 4, 371-393.

NAKamura, Y., OhYA, Y. \& Tsuruta, H. 1991 Experiments on vortex shedding from flat plates with square leading and trailing edges. J. Fluid Mech. 222, 437-447.

NAUdAscher, E. \& Rockwell, D. 1991 Hydrodaynamic forces. In IAHR Hydrodynamic Structures Design Manual, Vol.3. Balkena.

NAUdASCHER, E. \& AND RockWELl, D. 1993 Oscillator-model approach to the identification and assessement of flow-induced vibrations in a system. J. Hydraul. Res. 18, 59-82.

NAUdAscher, E. \& WANG, Y. 1993 Flow-induced vibrations of prismatic bodies and grids of prisms. J. Fluids Struct. 7, 341-373.

PARKer, R. \& Welsh, M. C. 1983 Effects of sound on flow separation from blunt flat plates. Intl J. Heat Fluid Flow 4 (2), 113-127.

RAMBERG, S. E. 1983 The effects of yaw and finite length upon the vortex wakes of stationary and vibrating circular cylinders. J. Fluid Mech. 128, 81-107.

Richter, A. \& NAUDASCHER, E. 1976 Fluctuating forces on a rigid circular cylinder in confined flow. J. Fluid Mech. 78, 561-576.

SARPKAYA, T. 1978 Fluid forces on oscillating cylinders. J. Waterway Port Coastal Ocean Div. WW4, 275-290.

Shiraishi, N. \& MAtsumoto, M. 1983 On classification of vortex-induced oscillation and its application for bridge structures. J. Wind Engng Ind. Aerodyn. 14, 419-430.

Williamson, C. H. K. \& BROWN, G. L. 1998 A series in $1 / \sqrt{R e}$ to represent the Strouhal-Reynolds number relationship of the cylinder wake. J. Fluids Struct. 12, 1073-1085.

Williamson, C. H. K. \& RoshKo, A. 1990 Measurements of base pressure in the wake of a cylinder at low Reynolds numbers. Z. Flugwiss. Weltraumforsch. 14, 38-46. 